\title{
Local regularity for an anisotropic elliptic equation
}

\author{
Naian Liao ${ }^{1} \cdot$ Igor I. Skrypnik ${ }^{2}$. Vincenzo Vespri ${ }^{3}$
}

Received: 21 November 2019 / Accepted: 30 April 2020 / Published online: 21 June 2020

(c) The Author(s) 2020

\section{Abstract}

We establish the interior Hölder continuity for locally bounded solutions, and the Harnack inequality for non-negative continuous solutions to a class of anisotropic elliptic equations with bounded and measurable coefficients, whose prototype equation is

$$
u_{x x}+\Delta_{q, y} u=0 \quad \text { locally in } \mathbb{R} \times \mathbb{R}^{N-1}, \text { for } q<2,
$$

via ideas and tools originating from the regularity theory for degenerate and singular parabolic equations.

Mathematics Subject Classification 35J70 $\cdot 35 \mathrm{~J} 92 \cdot 35 \mathrm{~B} 65$

\section{Introduction}

\subsection{Notation and the main results}

Let $E$ be an open set in $\mathbb{R}^{N}$ with $N \geq 2$. We denote a general point in $E$ by $z=(x, y) \in$ $\mathbb{R} \times \mathbb{R}^{N-1}$. For a function $u$ defined in $E$, the symbols $D_{x} u$ (or $u_{x}$ ) and $D_{y_{i}} u$ (or $u_{y_{i}}$ ) represent the differentiation of $u$ with respect to $x$ and $y_{i}$ variables. Accordingly we also set

$$
D_{y}=\left(D_{y_{1}}, \ldots, D_{y_{N-1}}\right), \quad D=\left(D_{x}, D_{y}\right) .
$$

Communicated by Y. Giga.

$凶 \quad$ Naian Liao

naian.liao@sbg.ac.at

Igor I. Skrypnik

iskrypnik@iamm.donbass.com

Vincenzo Vespri

vincenzo.vespri@unifi.it

1 Fachbereich Mathematik, Universität Salzburg, Hellbrunner Str. 34, 5020 Salzburg, Austria

2 Institute of Applied Mathematics and Mechanics, National Academy of Sciences of Ukraine, Gen. Batiouk Str. 19, Sloviansk 84116, Ukraine

3 Dipartimento di Matematica e Informatica "U. Dini”, Università degli Studi di Firenze, Via Morgagni 67/A, 50134 Florence, Italy 
For $1<q<2$, we shall consider the elliptic partial differential equation

$$
u_{x x}+\sum_{i=1}^{N-1} D_{y_{i}} A_{i}(z, u, D u)=0 \quad \text { weakly in } E,
$$

where the functions $A_{i}(z, u, \xi): E \times \mathbb{R} \times \mathbb{R}^{N} \rightarrow \mathbb{R}$ are Carathéodory functions, i.e. they are measurable in $(u, \xi)$ for all $z \in E$ and continuous in $z$ for a.e. $(u, \xi) \in \mathbb{R}^{N+1}$. Moreover, they are subject to the following structure conditions a.e. in $E$

$$
\left\{\begin{array}{l}
\sum_{i=1}^{N-1} A_{i}(z, u, D u) \cdot D_{y_{i}} u \geq C_{o}\left|D_{y} u\right|^{q} \\
\left|A_{i}(z, u, D u)\right| \leq C_{1}\left|D_{y} u\right|^{q-1} \quad i=1, \ldots, N-1
\end{array}\right.
$$

with given positive constants $C_{o}$ and $C_{1}$. The prototype equation is

$$
u_{x x}+\operatorname{div}_{y}\left(\left|D_{y} u\right|^{q-2} D_{y} u\right)=0 .
$$

Before stating the main result, let us recall that the anisotropic elliptic partial differential Eq. (1) is a special case of the more general equation

$$
\sum_{i=1}^{N} D_{x_{i}} A_{i}(x, u, D u)=0 \quad \text { weakly in } E,
$$

where the functions $A_{i}(x, u, \xi): E \times \mathbb{R} \times \mathbb{R}^{N} \rightarrow \mathbb{R}$ are Carathéodory functions, and subject to the structure conditions

$$
\left\{\begin{array}{l}
A_{i}(x, u, D u) \cdot u_{x_{i}} \geq C_{o}\left|u_{x_{i}}\right|^{p_{i}} \\
\left|A_{i}(x, u, D u)\right| \leq C_{1}\left|u_{x_{i}}\right|^{p_{i}-1}
\end{array}\right.
$$

for some constants $p_{i}>1, C_{o}>0$ and $C_{1}>0$. The prototype equation is

$$
\sum_{i=1}^{N}\left(a_{i}(x)\left|u_{x_{i}}\right|^{p_{i}-2} u_{x_{i}}\right)_{x_{i}}=0 \quad \text { weakly in } E .
$$

Here $a_{i}(x), i=1, \ldots, N$ are measurable functions, satisfying $C_{o} \leq a_{i}(x) \leq C_{1}$ for some positive $C_{o}$ and $C_{1}$. Note also we slightly abused the symbols $x$ and $D$ in (3) and (4), which represent a vector in $\mathbb{R}^{N}$ and the gradient in $x$.

When $p_{1}=\cdots=p_{N}=p>1$, the general Eq. (3) reduces to the standard $p$-Laplacian type equation whose local regularity theory, such as Hölder regularity and Harnack estimates, is well studied. See [3,13,21,22,24], for example.

When $p_{i}$ 's are potentially different, the study of local regularity was initiated in [17-20]. It has been shown that solutions are Lipschitz continuous, provided the coefficients of the operator are differentiable and satisfy some proper structure conditions. See also [14,15]. However, much less is known about the local regularity for (3) with merely bounded and measurable coefficients.

It has been proved that the local boundedness of local solutions to (3) is inherent in the notion of weak solution in $[10,12]$, provided

$$
\max \left\{p_{1}, \ldots, p_{N}\right\} \leq \frac{N \bar{p}}{N-\bar{p}} \quad \text { where } \quad \frac{1}{\bar{p}}=\frac{1}{N} \sum_{i=1}^{N} \frac{1}{p_{i}} .
$$


The above condition indicates that the indices $p_{i}$ 's are not too far apart. In fact, examples are constructed in $[11,17]$ to show that solutions could be unbounded, if the indices are too far apart. Recently, a rather detailed discussion on this issue has been carried out in [1].

Concerning the continuity of locally bounded solutions, a first step was made in [16]. It has been shown that locally bounded solutions to (1) are Hölder continuous for $q>2$. We also mention that the Hölder regularity for more general anisotropic operators is considered in $[8,9]$.

In order to define the notion of weak solution formally, we introduce the following anisotropic Sobolev spaces

$$
\begin{aligned}
& W^{1,[2, q]}(E)=\left\{u \in L^{1}(E): u_{x} \in L^{2}(E), u_{y_{i}} \in L^{q}(E), i=1, \ldots, N-1\right\}, \\
& W_{o}^{1,[2, q]}(E)=W_{o}^{1,1}(E) \cap W^{1,[2, q]}(E) .
\end{aligned}
$$

A function $u \in W_{\text {loc }}^{1,[2, q]}(E)$ is called a local, weak sub(super)-solution to (1), if for every compact set $K \subset E$ it satisfies

$$
\int_{K} u_{x} \phi_{x} d z+\int_{K} \sum_{i=1}^{N-1} A_{i}(z, u, D u) \phi_{y_{i}} d z \leq(\geq) 0,
$$

for all non-negative test functions $\phi \in W_{o}^{1,[2, q]}(K)$.

In this note, by sub(super)-solutions we always refer to the weak ones defined above. When we speak of structural data, we refer to the set of parameters $\left\{q, N, C_{o}, C_{1}\right\}$. We also write $\gamma$ as a generic positive constant that can be quantitatively determined a priori only in terms of the data and it may change line by line. When we write $\gamma(b)$ we mean to emphasize the dependence on the quantity $b$.

For $\rho>0$ let $K_{\rho}(y)$ be the cube of center at $y$ in $\mathbb{R}^{N-1}$ and edge $2 \rho$. When $y=0$ we simply write $K_{\rho}$. For $z_{o}=\left(x_{o}, y_{o}\right) \in E$, we define the cylinders scaled by a positive parameter $\theta$ :

$$
\begin{cases}\text { centered cylinders: } & z_{o}+Q_{\rho}(\theta)=\left[x_{o}-\theta \rho^{\frac{q}{2}}, x_{o}+\theta \rho^{\frac{q}{2}}\right] \times K_{\rho}\left(y_{o}\right), \\ \text { forward cylinders: } & z_{o}+Q_{\rho}^{+}(\theta)=\left[x_{o}, x_{o}+\theta \rho^{\frac{q}{2}}\right] \times K_{\rho}\left(y_{o}\right), \\ \text { backward cylinders: } & z_{o}+Q_{\rho}^{-}(\theta)=\left[x_{o}-\theta \rho^{\frac{q}{2}}, x_{o}\right] \times K_{\rho}\left(y_{o}\right) .\end{cases}
$$

Concerning the parameter $\theta$, it will be a quantity that restores the homogeneity of the Eq. (1) depending on the structure it is subject to. More precisely, we will set $\theta=[u]^{\frac{2-q}{2}}$, where $[u]$ represents a quantity that is of the "dimension" of a given solution $u$. When $q=2$, the cylinder $z_{o}+Q_{\rho}(\theta)$ formally recovers the standard cube in $\mathbb{R}^{N}$ of center at $z_{o}$ and edge $2 \rho$. The exact form of $\theta$ hinges upon the context and will be specified in the following. Thus, such cylinders are intrinsically scaled. When $\theta=1$, we simply write $z_{o}+Q_{\rho}^{ \pm}$.

Suppose that $u \in L^{\infty}(E)$ is a local weak solution to (1) and (2). For a compact set $K \subset E$ introduce the intrinsic distance from $K$ to $\partial E$ by

$$
(2, q)-\operatorname{dist}(K ; \partial E) \stackrel{\operatorname{def}}{=} \inf _{\substack{z \in K \\ \bar{z} \in \partial E}}\left\{|y-\bar{y}|+\|u\|_{\infty, E}^{\frac{q-2}{q}}|x-\bar{x}|^{\frac{2}{q}}\right\} .
$$

Fix $z_{o} \in E$ and let $R>0$ be so small that

$$
Q_{o} \stackrel{\text { def }}{=}\left[x_{o}-R^{\frac{q}{2}}, x_{o}+R^{\frac{q}{2}}\right] \times K_{R}\left(y_{o}\right) \subset E
$$


for a small parameter $\varepsilon>0$; we may assume that $z_{o}$ coincides with the origin. We set

$$
\mu^{+}=\underset{Q_{o}}{\operatorname{ess} \sup u,} \quad \mu^{-}=\underset{Q_{o}}{\operatorname{ess} \inf u,} \quad \omega=\underset{Q_{o}}{\operatorname{ess} \operatorname{osc}} u=\mu^{+}-\mu^{-} .
$$

Construct the cylinder

$$
Q_{R}(\theta)=\left[-\theta R^{\frac{q}{2}}, \theta R^{\frac{q}{2}}\right] \times K_{R} \quad \text { with } \quad \theta=\left(\frac{\omega}{\Lambda}\right)^{\frac{2-q}{2}},
$$

where $\Lambda>1$ is a parameter to be determined in terms of the data only. Without loss of generality, we may assume that $\omega \leq 1$, such that

$$
Q_{R}(\theta) \subset Q_{o} \text { and } \underset{Q_{R}(\theta)}{\operatorname{ess} \operatorname{osc}} u \leq \omega .
$$

This $Q_{R}(\theta)$ is the starting cylinder for the reduction of oscillation.

Now we state our theorem concerning the interior Hölder regularity which holds for all $1<q<2$.

Theorem 1 Let $u$ be a bounded, local, weak solution to (1) and (2) in E. Then $u$ is locally Hölder continuous in E. More precisely, there exist constants $\gamma, \Lambda>1$ and $\alpha \in(0,1)$ that can be determined a priori only in terms of the data, such that for any $0<\rho<R$, we have

$$
\underset{z_{o}+Q_{\rho}(\theta)}{\operatorname{ess} O s c} u \leq \gamma \omega\left(\frac{\rho}{R}\right)^{\alpha} .
$$

In other words, for every compact set $K \subset E$,

$$
\left|u\left(z_{1}\right)-u\left(z_{2}\right)\right| \leq \gamma\|u\|_{\infty, E}\left(\frac{\left|y_{1}-y_{2}\right|+\|u\|_{\infty, E}^{\frac{q-2}{q}}\left|x_{1}-x_{2}\right|^{\frac{2}{q}}}{(2, q)-\operatorname{dist}(K ; \partial E)}\right)^{\alpha}
$$

for every pair of points $z_{1}, z_{2} \in K$.

In order to state Harnack's inequality, introduce the following intrinsically scaled cylinder centered at $z_{o} \in E$ :

$$
z_{o}+Q_{4 \rho}(\theta) \quad \text { where } \theta=\left[\bar{c} u\left(z_{o}\right)\right]^{\frac{2-q}{2}},
$$

where $\bar{c} \in(0,1)$ will be determined in terms of the data and

$$
\frac{2(N-1)}{N} \stackrel{\text { def }}{=} q_{*}<q<2 .
$$

It is worth mentioning that (6) ensures $\kappa \stackrel{\text { def }}{=} N q-2(N-1)>0$.

Since every locally bounded, local, weak solution to (1) and (2) has a continuous representative by Theorem 1, we may only deal with continuous weak solutions.

Theorem 2 Let $u$ be a continuous, non-negative, local, weak solution to (1) and (2) in E. Assume (6) holds and the cylinder (5) is contained in E. There exist constants $c, \bar{c} \in(0,1)$ depending only on the data, such that

$$
c \sup _{z_{o}+Q_{\rho}(\theta)} u \leq u\left(z_{o}\right) \leq c^{-1} \inf _{z_{o}+Q_{\rho}(\theta)} u .
$$


Theorem 2 has been stated for continuous solutions, to give meaning to $u\left(z_{o}\right)$. However, it continues to hold for non-negative weak solutions to (1) and (2) for almost all $z_{o} \in E$ and for the corresponding intrinsic cylinders.

The proofs of Theorems 1 and 2 are based on the following expansion of positivity. To this end, we introduce another intrinsically scaled cylinder centered at $z_{o} \in E$ :

$$
z_{o}+Q_{16 \rho}(\theta) \quad \text { where } \theta=\delta M^{\frac{2-q}{2}},
$$

for some positive number $M$ and a parameter $\delta \in(0,1)$ to be determined in terms of the data only.

Proposition 1 Let u be a non-negative, local, weak super-solution to (1) and (2) in E. Assume the cylinder (7) is contained in $E$ and

$$
\left|\left[u\left(x_{o}, \cdot\right) \geq M\right] \cap K_{\rho}\left(y_{o}\right)\right| \geq \beta\left|K_{\rho}\right|
$$

for some $\beta \in(0,1)$ and $M>0$. There exist constants $\eta, \delta \in(0,1)$ depending only upon the data and $\beta$, such that

$$
u \geq \eta M \quad \text { a.e. in } z_{o}+Q_{2 \rho}(\theta) \quad \text { with } \theta=\delta M^{\frac{2-q}{2}} .
$$

The proof of Theorem 2 will also use the following $L_{\mathrm{loc}}^{1}-L_{\mathrm{loc}}^{\infty}$ estimate.

Theorem 3 Let $u$ be a non-negative, local, weak solution to (1) and (2) in E and assume (6) holds. There exists a constant $\gamma>0$ depending only on the data, such that for all cylinders

$$
\left[x_{o}-2 t, x_{o}+2 t\right] \times K_{2 \rho}\left(y_{o}\right) \subset E,
$$

we have

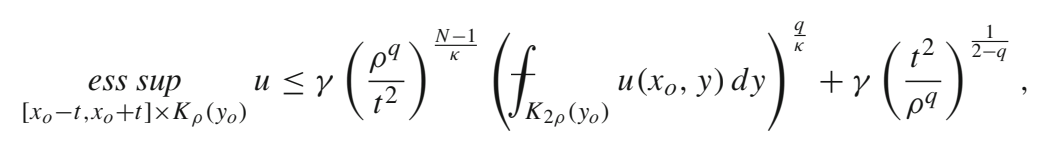

where $\kappa=N q-2(N-1)$.

Remark 1 Our main results continue to hold if we multiply $u_{x x}$ in (1) by a measurable coefficient $a(y)$ satisfying $C_{o} \leq a(y) \leq C_{1}$ for some positive $C_{o}$ and $C_{1}$. Nevertheless we do not know how to demonstrate them for more general operators at this moment. See (8)-(10) for the technical obstruction.

\subsection{Novelty and significance}

The main contribution of this work is to present a local regularity theory regarding (1) in the case $q<2$. In Theorem 1, we obtain that locally bounded solutions are Hölder continuous for all $1<q<2$. Moreover, we establish an intrinsic Harnack inequality for non-negative solutions to (1) in Theorem 2, which holds only for $q>q_{*}$, where $q_{*}>1$ is defined in (6). The main tool of proving Theorems 1 and 2, i.e. the expansion of positivity for non-negative, super-solutions, is presented in Proposition 1. Such a property plays a central role in any kind of Harnack estimates for elliptic and parabolic equations. Moreover, Theorem 3 serves as another main component in the proof of Theorem 2. It may be viewed as a Harnack inequality in $L_{\text {loc }}^{1}-L_{\text {loc }}^{\infty}$ topology.

The main idea of treating the local regularity issues for (1) is the so-called intrinsic scaling, which was originally formulated in the theory of degenerate and singular parabolic 
equations. We refer to $[2,4,7]$ for an account of the theory. To realize such a key idea, we also owe technical tools to the parabolic theory. The exponential shift technique in our proof of Proposition 1 is borrowed from [6], which was used to handle the singular parabolic equations. Theorem 3 also has a counterpart in the theory of singular parabolic equations, though we need some new input to prove it. See [5] and [7, Appendix A]. However, it is not immediately clear whether the same kind of approach can be applied to more general anisotropic $p$-Laplacian operators. We think this intriguing interplay between the theories of elliptic equations and parabolic equations deserves a deeper understanding in the future investigation.

As for the organization of this note, we first collect some preliminary tools in Sect. 2, including energy estimates, DeGiorgi-type lemmas, a logarithmic lemma and its consequences. In Sect. 3, we give a proof of Proposition 1, concerning the expansion of positivity. Section 4 is devoted to the proof of Theorem 1. To streamline the presentation, we show Theorem 2 in Sect. 5 assuming Theorem 3, which will be proven in Sect. 6.

\section{Preliminaries}

For a multi-index $\mathbf{p}=\left(p_{1}, \ldots, p_{N}\right), p_{i} \geq 1$, introduce anisotropic Sobolev spaces

$$
\begin{aligned}
& W^{1, \mathbf{p}}(E)=\left\{u \in L^{1}(E): u_{x_{i}} \in L^{p_{i}}(E), i=1, \ldots, N\right\}, \\
& W_{o}^{1, \mathbf{p}}(E)=W_{o}^{1,1}(E) \cap W^{1, \mathbf{p}}(E) .
\end{aligned}
$$

We state here the Sobolev-Troisi inequality [23].

Lemma 1 Let $E \subset \mathbb{R}^{N}$ be a bounded open set and consider $u \in W_{o}^{1, \mathbf{p}}(E), p_{i} \geq 1$ for all $i=1, \ldots, N$. Set

$$
\frac{1}{\bar{p}}=\frac{1}{N} \sum_{i=1}^{N} \frac{1}{p_{i}}, \quad \bar{p}^{*}=\frac{N \bar{p}}{N-\bar{p}} .
$$

If $\bar{p}<N$, then there exists a positive constant $\gamma$ depending only on the set of parameters $\{N, \mathbf{p}\}$, such that

$$
\|u\|_{\bar{p}^{*}}^{N} \leq \gamma \prod_{i=1}^{N}\left\|u_{x_{i}}\right\|_{p_{i}} .
$$

According to our notion of weak solution for (1), the index we will use is $\mathbf{p}=(2, q, \ldots, q)$.

\subsection{Energy estimates}

For $k \in \mathbb{R}$, we set

$$
(u-k)_{+}=\max \{u-k, 0\}, \quad(u-k)_{-}=\max \{-(u-k), 0\} .
$$

The first proposition concerns energy estimates in the interior.

Proposition 2 Let $u$ be a local, weak sub(super)-solution to (1) and (2) in E. Then there exists a constant $\gamma>0$ depending only on the data, such that for any cube $Q \subset E$ and for 
every non-negative, piecewise smooth cutoff function $\zeta$ vanishing on $\partial Q$, we have for any $k \in \mathbb{R}$ that

$$
\begin{aligned}
& \int_{Q}\left|D_{x}(u-k)_{ \pm}\right|^{2} \zeta^{2} d z+\int_{Q}\left|D_{y}(u-k)_{ \pm}\right|^{q} \zeta^{2} d z \\
& \quad \leq \gamma \int_{Q}(u-k)_{ \pm}^{2}\left|\zeta_{x}\right|^{2} d z+\gamma \int_{Q}(u-k)_{ \pm}^{q}\left|D_{y} \zeta\right|^{q} d z .
\end{aligned}
$$

Proof We only show the case of super-solutions, the other case being similar. In the weak formulation of (1), we take the test function $-(u-k)-\zeta^{2}$; a standard calculation yields that

$$
\begin{aligned}
& -\int_{Q} \zeta^{2} u_{x} D_{x}(u-k)_{-} d z-\int_{Q} \zeta^{2} \sum_{i=1}^{N-1} A_{i}(z, u, D u) D_{y_{i}}(u-k)_{-} d z \\
& \quad \leq 2 \int_{Q} \zeta\left|u_{x}\right|(u-k)_{-}\left|\zeta_{x}\right| d z+2 \int_{Q} \zeta \sum_{i=1}^{N-1}\left|A_{i}(z, u, D u)\right|(u-k)_{-}\left|D_{y_{i}} \zeta\right| d z
\end{aligned}
$$

We will employ the structure conditions and Young's inequality repeatedly in estimating the various terms. We first estimate the terms on the left-hand side. The first term is

$$
-\int_{Q} \zeta^{2} u_{x} D_{x}(u-k)_{-} d z=\int_{Q}\left|D_{x}(u-k)_{-}\right|^{2} \zeta^{2} d z
$$

while the second term is estimated by

$$
-\int_{Q} \zeta^{2} \sum_{i=1}^{N-1} A_{i}(z, u, D u) D_{y_{i}}(u-k)_{-} d z \geq C_{o} \int_{Q}\left|D_{y}(u-k)_{-}\right|^{q} \zeta^{2} d z .
$$

Now we estimate the terms on the right-hand side. The first term is estimated by

$$
2 \int_{Q} \zeta\left|u_{x}\right|(u-k)_{-}\left|\zeta_{x}\right| d z \leq \frac{1}{2} \int_{Q} \zeta^{2}\left|D_{x}(u-k)_{-}\right|^{2} d z+2 \int_{Q}(u-k)_{-}^{2}\left|\zeta_{x}\right|^{2} d z .
$$

Similarly, the second term is estimated by

$$
\begin{aligned}
& 2 \int_{Q} \zeta \sum_{i=1}^{N-1}\left|A_{i}(z, u, D u)\right|(u-k)_{-}\left|D_{y_{i}} \zeta\right| d z \\
& \quad \leq \frac{C_{o}}{2} \int_{Q} \zeta^{2}\left|D_{y}(u-k)_{-}\right|^{q} d z+\gamma \int_{Q}(u-k)_{-}^{q}\left|D_{y} \zeta\right|^{q} d z .
\end{aligned}
$$

Collecting all these estimate gives the desired result.

The second proposition concerns energy estimates involving boundary information. We state it in the case of super-solutions, while sub-solutions have an analogous statement. Let $\theta$ and $M$ be positive parameters.

Proposition 3 Let u be a local, weak super-solution to (1) and (2) in E. Suppose $z_{o}+Q_{\rho}^{ \pm}(\theta) \subset$ $E$ and

$$
u\left(x_{o}, \cdot\right) \geq M \quad \text { a.e. in } K_{\rho}\left(y_{o}\right) .
$$

Then there exists a constant $\gamma>0$ depending only on the data, such that for any $k \leq M$ and for every non-negative, piecewise smooth cutoff function $\zeta$ vanishing on $\partial Q_{\rho}(\theta)$, we have

$$
\int_{z_{o}+Q_{\rho}^{ \pm}(\theta)}\left|D_{x}(u-k)_{-}\right|^{2} \zeta^{2} d z+\int_{z_{o}+Q_{\rho}^{ \pm}(\theta)}\left|D_{y}(u-k)_{-}\right|^{q} \zeta^{2} d z
$$




$$
\leq \gamma \int_{z_{o}+Q_{\rho}^{ \pm}(\theta)}(u-k)_{-}^{2}\left|\zeta_{x}\right|^{2} d z+\gamma \int_{z_{o}+Q_{\rho}^{ \pm}(\theta)}(u-k)_{-}^{q}\left|D_{y} \zeta\right|^{q} d z
$$

Proof Assume $z_{o}=(0,0)$. The function $-(u-k)-\zeta^{2}$ vanishes on the boundary of $Q_{\rho}^{ \pm}(\theta)$, since $u(0, \cdot) \geq M$ on the set $K_{\rho}$ and $k \leq M$, and thus it is an admissible test function. Using this test function in the weak formulation in $Q_{\rho}^{ \pm}(\theta)$, the remaining calculation runs similar to the proof of Proposition 2.

\subsection{DeGiorgi-type Lemmas}

Suppose $\theta$ and $M$ are positive parameters.

Lemma 2 Let $u$ be a non-negative, local, weak super-solution to (1) and (2) in E. There exists a number $v>0$ depending only upon the parameters $\theta, M$ and the data, such that if

$$
\left|[u \leq M] \cap\left[z_{o}+Q_{2 \rho}(\theta)\right]\right| \leq v\left|Q_{2 \rho}(\theta)\right|,
$$

then

$$
u \geq \frac{M}{2} \quad \text { a.e. in } z_{o}+Q_{\rho}(\theta)
$$

Proof We may assume $z_{o}=(0,0)$. For $n=0,1, \ldots$, we set

$$
\begin{aligned}
k_{n} & =\frac{M}{2}+\frac{M}{2^{n+1}}, \quad \rho_{n}=\rho+\frac{\rho}{2^{n}}, \quad \tilde{\rho}_{n}=\frac{\rho_{n}+\rho_{n+1}}{2}, \\
Q_{n} & =Q_{\rho_{n}}(\theta), \quad \tilde{Q}_{n}=Q_{\tilde{\rho}_{n}}(\theta) .
\end{aligned}
$$

Introduce the cutoff function $\zeta$ vanishing on $\partial Q_{n}$ and equal to identity in $\tilde{Q}_{n}$, such that

$$
\left|D_{y} \zeta\right| \leq \gamma \frac{2^{n}}{\rho} \quad \text { and } \quad\left|\zeta_{x}\right| \leq \frac{\gamma}{\theta}\left(\frac{2^{n}}{\rho}\right)^{\frac{q}{2}} .
$$

In this setting the energy estimate in Proposition 2 yields

$$
\begin{aligned}
& \int_{\tilde{Q}_{n}}\left|D_{x}\left(u-k_{n}\right)_{-}\right|^{2} d z+\int_{\tilde{Q}_{n}}\left|D_{y}\left(u-k_{n}\right)_{-}\right|^{q} d z \\
& \leq \gamma \frac{2^{q n}}{\theta^{2} \rho^{q}} \int_{Q_{n}}\left(u-k_{n}\right)_{-}^{2} d z+\gamma \frac{2^{q n}}{\rho^{q}} \int_{Q_{n}}\left(u-k_{n}\right)_{-}^{q} d z \\
& \leq \gamma \frac{2^{q n} M^{q}}{\rho^{q}}\left[1+\frac{M^{2-q}}{\theta^{2}}\right]\left|A_{n}\right|,
\end{aligned}
$$

where we have set $A_{n}=\left[u<k_{n}\right] \cap Q_{n}$. Now setting $\zeta$ to be a cutoff function which vanishes on the boundary of $\tilde{Q}_{n}$ and equals identity in $Q_{n+1}$, an application of Lemma 1 gives that

$$
\begin{aligned}
\left(\frac{M}{2^{n+2}}\right)^{\bar{q}}\left|A_{n+1}\right| & \leq \int_{\tilde{Q}_{n}}\left(u-k_{n}\right)_{-}^{\bar{q}} \zeta^{\bar{q}} d z \\
& \leq\left(\int_{\tilde{Q}_{n}}\left[\left(u-k_{n}\right)_{-} \zeta\right]^{\frac{N \bar{q}}{N-\bar{q}}} d z\right)^{\frac{N-\bar{q}}{N}}\left|A_{n}\right|^{\frac{\bar{q}}{N}} \\
& \leq \gamma\left(\left\|D_{x}\left(u-k_{n}\right)_{-}\right\|_{2} \prod_{i=1}^{N-1}\left\|D_{y_{i}}\left(u-k_{n}\right)_{-}\right\|_{q}\right)^{\frac{\bar{q}}{N}}\left|A_{n}\right|^{\frac{\bar{q}}{N}}
\end{aligned}
$$




$$
\leq \gamma \frac{2^{q n} M^{q}}{\rho^{q}}\left(1+\frac{M^{2-q}}{\theta^{2}}\right)\left|A_{n}\right|^{1+\frac{\bar{q}}{N}},
$$

where $\bar{q}$ satisfies

$$
\frac{1}{\bar{q}}=\frac{1}{N}\left(\frac{1}{2}+\frac{N-1}{q}\right)
$$

In terms of $Y_{n}=\left|A_{n}\right| /\left|Q_{n}\right|$, this can be rewritten as

$$
Y_{n+1} \leq \gamma \gamma_{1}^{n}\left(\frac{\theta^{2}}{M^{2-q}}\right)^{\frac{\bar{q}}{2 N}}\left(1+\frac{M^{2-q}}{\theta^{2}}\right) Y_{n}^{1+\frac{\bar{q}}{N}},
$$

where $\gamma_{1}$ and $\gamma$ depend only on the data. Hence by [2, Chapter I, Lemma 4.1], $Y_{n} \rightarrow 0$ if we require that

$$
Y_{o} \leq \frac{1}{\gamma}\left(\frac{M^{2-q}}{\theta^{2}}\right)^{\frac{1}{2}}\left(1+\frac{M^{2-q}}{\theta^{2}}\right)^{-\frac{N}{\bar{q}}} \stackrel{\text { def }}{=} v .
$$

We also have a version involving boundary data.

Lemma 3 Let $u$ be a non-negative, local weak super-solution to (1) and (2) in E. Suppose $z_{o}+Q_{2 \rho}^{ \pm}(\theta) \subset E$ and $u\left(x_{o}, \cdot\right) \geq M$ on the set $K_{2 \rho}\left(y_{o}\right)$. There exists a number $v>0$ depending only upon the parameters $\theta, M$ and the data, such that if

$$
\left|[u \leq M] \cap\left[z_{o}+Q_{2 \rho}^{ \pm}(\theta)\right]\right| \leq v\left|Q_{2 \rho}^{ \pm}(\theta)\right|,
$$

then

$$
u(x, y) \geq \frac{M}{2} \quad \text { a.e. in } z_{o}+Q_{\rho}^{ \pm}(\theta) .
$$

Proof The proof is similar to that of Lemma 2. This time, we may employ the energy estimate in Proposition 3, since $k_{n} \leq M$. The constant $v$ presents the same form as in Lemma 2.

\subsection{A logarithmic estimate}

Let $Q$ be a cylinder in $E$. Suppose $u$ is a non-negative, weak super-solution to (1) and (2) in $E$. For $a \in(0,1)$ and $M>0$, we introduce the following function in $Q$ :

$$
G(u(z))=\left[\frac{1}{u(z)+a M}-\frac{1}{M}\right]_{+} .
$$

Note that $G(u)=0$ if $u \geq(1-a) M \stackrel{\text { def }}{=} k$.

Lemma 4 Let $u$ be a non-negative, local, weak super-solution to (1) and (2) in E. There exists a constant $\gamma>0$ depending only on the data, such that for every piecewise smooth cutoff function $\zeta$ in $Q$ vanishing on $\partial Q$, there holds

$$
\begin{aligned}
& \int_{Q}\left|D_{x} \ln _{+}\left(\frac{M}{u+a M}\right)\right|^{2} \zeta^{2} d z \\
& \quad \leq \gamma \int_{Q \cap[u<k]}\left|D_{y} \zeta\right|^{q}(u+a M)^{q-2} d z+\gamma \int_{Q \cap[u<k]}\left|\zeta_{x}\right|^{2} d z .
\end{aligned}
$$


Proof Use the test function $\phi=-G(u) \zeta^{2} \in W_{o}^{1,[2, q]}$ in the weak formulation of $u$ to get

$$
\begin{gathered}
\int_{Q \cap[u<k]} u_{x} \frac{\zeta^{2} u_{x}}{(u+a M)^{2}} d z+\int_{Q \cap[u<k]} \sum_{i=1}^{N-1} A_{i}(z, u, D u) \frac{\zeta^{2} D_{y_{i}} u}{(u+a M)^{2}} d z \\
\leq 2 \int_{Q} \zeta \zeta_{x} u_{x} G(u) d z+2 \int_{Q} \zeta G(u) \sum_{i=1}^{N-1} A_{i}(z, u, D u) D_{y_{i}} \zeta d z .
\end{gathered}
$$

We use the structure conditions (2) and Young's inequality repeatedly to estimate various terms separately. For the left-hand side, the first term is

$$
\int_{Q \cap[u<k]}\left|u_{x}\right|^{2} \frac{\zeta^{2}}{(u+a M)^{2}} d z=\int_{Q}\left|D_{x} \ln _{+}\left(\frac{M}{u+a M}\right)\right|^{2} \zeta^{2} d z .
$$

The second term on the left is treated similarly:

$$
\int_{Q \cap[u<k]} A_{i}(z, u, D u) \frac{\zeta^{2} D_{y_{i}} u}{(u+a M)^{2}} d z \geq C_{o} \int_{Q} \frac{\left|D_{y} u\right|^{q} \zeta^{2} \chi_{[u<k]}}{(u+a M)^{2}} d z .
$$

For the right-hand side, the first term is estimated by

$$
\begin{aligned}
& 2 \int_{Q} \zeta \zeta_{x} u_{x} G(u) d z \leq 2 \int_{Q} \zeta\left|\zeta_{x}\right| G(u)\left|u_{x}\right| d z \\
& \quad \leq 2 \int_{Q}\left|D_{x} \ln _{+}\left(\frac{M}{u+a M}\right)\right| \zeta\left|\zeta_{x}\right| d z \\
& \quad \leq \frac{1}{2} \int_{Q}\left|D_{x} \ln _{+}\left(\frac{M}{u+a M}\right)\right|^{2} \zeta^{2} d z+2 \int_{Q}\left|\zeta_{x}\right|^{2} \chi_{[u<k]} d z .
\end{aligned}
$$

The second term is estimated similarly:

$$
\begin{aligned}
& 2 \int_{Q} \zeta G(u) \sum_{i=1}^{N-1} A_{i}(z, u, D u) D_{y_{i}} \zeta d z \leq \frac{C_{o}}{4} \int_{Q} \frac{\left|D_{y} u\right|^{q} \zeta^{2} \chi[u<k]}{(u+a M)^{2}} d z \\
& \quad+\gamma \int_{Q \cap[u<k]}\left|D_{y} \zeta\right|^{q}(u+a M)^{q-2} d z .
\end{aligned}
$$

Collecting all above estimates yields the desired result.

\subsection{Consequences of the logarithmic estimate}

Suppose $u$ is a non-negative, local, weak super-solution to (1) and (2) in $E$. Let $z_{o} \in E$, $\rho>0$ and $M>0$, such that $z_{o}+Q_{2 \rho}(\theta) \subset E$, where for some $j_{*}>0$ to be determined we have defined

$$
\theta=\left(2^{-j_{*}} M\right)^{\frac{2-q}{2}}
$$

\subsubsection{Propagation of measure theoretical information}

Lemma 5 Assume for some $\beta \in(0,1)$ there holds

$$
\left|\left[u\left(x_{o}, \cdot\right) \geq M\right] \cap K_{\rho}\left(y_{o}\right)\right| \geq \beta\left|K_{\rho}\right| .
$$


Then there exists $j_{*}>0$ depending only on the data and $\beta$, such that

$$
\left|\left[u(x, \cdot) \geq \frac{M}{2^{j_{*}}}\right] \cap K_{\rho}\left(y_{o}\right)\right| \geq \frac{\beta}{2}\left|K_{\rho}\right| \text { for all } x \in\left[x_{o}-\theta \rho^{\frac{q}{2}}, x_{o}+\theta \rho^{\frac{q}{2}}\right] .
$$

Proof Assume $z_{o}=(0,0)$. We first apply Lemma 4 in $Q_{2 \rho}(\theta)$, choosing $a=2^{-j}$ for some $j \in\left\{1,2, \ldots, j_{*}\right\}$ and a cutoff function $\zeta$ that equals 1 in $Q_{\rho}(\theta)$ and vanishes on $\partial Q_{2 \rho}(\theta)$, such that

$$
\left|\zeta_{x}\right| \leq \frac{\gamma}{\theta \rho^{\frac{q}{2}}}, \quad\left|D_{y} \zeta\right| \leq \frac{\gamma}{\rho}
$$

In this way, we obtain

$$
\begin{aligned}
& \int_{Q_{\rho}(\theta)}\left|D_{x} \ln _{+}\left(\frac{M}{u+a M}\right)\right|^{2} d z \\
& \quad \leq \gamma \int_{Q_{2 \rho}(\theta)}\left|D_{y} \zeta\right|^{q}(u+a M)^{q-2} \chi_{[u<(1-a) M]} d z+\gamma \int_{Q_{2 \rho}(\theta)}\left|\zeta_{x}\right|^{2} d z \\
& \leq \frac{\gamma}{\theta^{2} \rho^{q}}\left|Q_{\rho}(\theta)\right| .
\end{aligned}
$$

From this, a straightforward application of Hölder's inequality yields

$$
\int_{Q_{\rho}(\theta)}\left|\psi_{x}\right| d z \leq \frac{\gamma}{\theta \rho^{\frac{q}{2}}}\left|Q_{\rho}(\theta)\right|,
$$

where, for ease of notation, we have set

$$
\psi(x, y)=\ln _{+}\left[\frac{M}{u(x, y)+a M}\right] .
$$

By the measure theoretical information known at $x=0$, we estimate

$$
\int_{K_{\rho}} \psi(0, y) d y \leq(1-\beta) j \ln 2 .
$$

These estimates joint with the mean value theorem give us

$$
\begin{aligned}
\int_{K_{\rho}} \psi(x, y) d y & \leq \int_{K_{\rho}} \psi(0, y) d y+\int_{K_{\rho}} \int_{-\theta \rho^{\frac{q}{2}}}^{\theta \rho^{\frac{q}{2}}}\left|\psi_{x}\right| d x d y \\
& \leq(1-\beta) j \ln 2+\frac{\gamma}{\theta \rho^{\frac{q}{2}}}\left|Q_{\rho}(\theta)\right| \\
& \leq(1-\beta) j \ln 2+\gamma\left|K_{\rho}\right|
\end{aligned}
$$

for all $x \in\left[-\theta \rho^{\frac{q}{2}}, \theta \rho^{\frac{q}{2}}\right]$. The left-hand side is estimated from below over a smaller set

$$
\left[u(x, \cdot) \leq \frac{M}{2^{j}}\right] \cap K_{\rho}
$$

to obtain

$$
\int_{K_{\rho}} \psi(x, y) d y \geq(j-1) \ln 2\left|\left[u(x, \cdot) \leq \frac{M}{2^{j}}\right] \cap K_{\rho}\right| .
$$


Collecting the above estimates, we have for all $x \in\left[-\theta \rho^{\frac{q}{2}}, \theta \rho^{\frac{q}{2}}\right]$,

$$
\left|\left[u(x, \cdot) \leq \frac{M}{2^{j}}\right] \cap K_{\rho}\right| \leq \frac{j}{j-1}(1-\beta)\left|K_{\rho}\right|+\frac{\gamma}{j-1}\left|K_{\rho}\right| .
$$

To conclude, we may choose $j_{*}$ large enough, such that

$$
\frac{\gamma}{j_{*}-1} \leq \frac{\beta}{4}, \quad \frac{j_{*}}{j_{*}-1}(1-\beta) \leq 1-\frac{3}{4} \beta
$$

\subsubsection{A shrinking lemma}

\section{Lemma 6 Assume}

$$
u\left(x_{o}, \cdot\right) \geq M \quad \text { a.e. in } K_{\rho}\left(y_{o}\right) .
$$

Then there exists a constant $\gamma>0$ depending only on the data, such that for any $j_{*}>0$

$$
\left|\left[u(x, \cdot) \leq \frac{M}{2^{j_{*}}}\right] \cap K_{\rho}\left(y_{o}\right)\right| \leq \frac{\gamma}{j_{*}}\left|K_{\rho}\right|,
$$

for all $x \in\left[x_{o}-\theta \rho^{\frac{q}{2}}, x_{o}+\theta \rho^{\frac{q}{2}}\right]$.

Proof Assume $z_{o}=(0,0)$. The proof is similar to that of Lemma 5. However, the quantitative information $u(0, \cdot) \geq M$ in $K_{\rho}$ yields

$$
\int_{K_{\rho}} \psi(0, y) d y=0 .
$$

Hence we have for all $x \in\left[-\theta \rho^{\frac{q}{2}}, \theta \rho^{\frac{q}{2}}\right]$,

$$
\left|\left[u(x, \cdot) \leq \frac{M}{2^{j}}\right] \cap K_{\rho}\right| \leq \frac{\gamma}{j-1}\left|K_{\rho}\right| .
$$

The proof is concluded by redefining $\gamma$ properly.

\subsubsection{Propagation of pointwise information}

A combination of Lemmas 3 and 6 shows that the positivity of a non-negative, local supersolution at $x_{o}$ over a $y$-cube spreads in $x$ direction over a smaller $y$-cube.

Lemma 7 Assume

$$
u\left(x_{o}, \cdot\right) \geq M \quad \text { a.e. in } K_{2 \rho}\left(y_{o}\right) .
$$

Then there exists $j_{*}>0$ depending only on the data, such that

$$
u(x, \cdot) \geq \frac{M}{2^{j_{*}+1}} \quad \text { a.e. in } K_{\rho}\left(y_{o}\right),
$$

for all $x \in\left[x_{o}-\theta \rho^{\frac{q}{2}}, x_{o}+\theta \rho^{\frac{q}{2}}\right]$ where $\theta=\left(2^{-j_{*}} M\right)^{\frac{2-q}{2}}$. 
Proof Assume $z_{o}=(0,0)$. By Lemma 3 in the cylinder $Q_{2 \rho}^{ \pm}(\theta)$ with $\theta=\left(2^{-j_{*}} M\right)^{\frac{2-q}{2}}$ for some $j_{*}>0$ to be chosen and $a=\frac{1}{2}$, there exists a positive constant $v$ depending only on the data, such that if

$$
\left|\left[u \leq \frac{M}{2^{j_{*}}}\right] \cap Q_{2 \rho}^{ \pm}(\theta)\right| \leq v\left|Q_{2 \rho}^{ \pm}(\theta)\right|
$$

then

$$
u \geq \frac{M}{2^{j_{*}+1}} \quad \text { a.e. in } Q_{\rho}^{ \pm}(\theta) .
$$

Now we choose the constant $j_{*}$ according to Lemma 6 , such that $\gamma j_{*}^{-1} \leq v$.

\section{Proof of Proposition 1}

The proof of Proposition 1 hinges upon the following preliminary version.

Proposition 4 Suppose the hypothesis in Proposition 1 holds. If for some $\beta \in(0,1)$ and $M>0$ there holds

$$
\left|[u(x, \cdot) \geq M] \cap K_{\rho}\left(y_{o}\right)\right| \geq \beta\left|K_{\rho}\right|
$$

for all

$$
\left|x-x_{o}\right| \leq M^{\frac{2-q}{2}} \rho^{\frac{q}{2}} .
$$

Then there exist constants $\eta, \delta \in(0,1)$ depending only upon the data and $\beta$, such that

$$
u(x, \cdot) \geq \eta M \quad \text { a.e. in } K_{2 \rho}\left(y_{o}\right)
$$

for all

$$
(1-\delta) M^{\frac{2-q}{2}} \rho^{\frac{q}{2}} \leq\left|x-x_{o}\right| \leq M^{\frac{2-q}{2}} \rho^{\frac{q}{2}} .
$$

We will assume without loss of generality that $z_{o}$ coincides with the origin. To simplify the presentation, we deal with the half space $[x>0]$ only, since the case of $[x<0]$ is similar.

\subsection{Change of variables}

Introduce the new variable $\tilde{z}=(\tilde{x}, \tilde{y})$ and the unknown function $v$ defined by

$$
\tilde{y}=\frac{y}{\rho}, \quad-e^{-\tilde{x}}=\frac{x-M^{\frac{2-q}{2}} \rho^{\frac{q}{2}}}{M^{\frac{2-q}{2}} \rho^{\frac{q}{2}}}, \quad v(\tilde{z})=\frac{1}{M} u(z) e^{\frac{2}{2-q} \tilde{x}} .
$$

It maps the cylinder $\left[0, M^{\frac{2-q}{2}} \rho^{\frac{q}{2}}\right] \times K_{16 \rho}$ into $(0, \infty) \times K_{16}$. Now we proceed to show that $v$ satisfies a differential inequality with a similar type of structure conditions as (2). To start with, we have by differentiation that

$$
u_{x}=\frac{M e^{(1-\lambda) \tilde{x}}}{M^{\frac{1}{\lambda}} \rho^{\frac{q}{2}}}\left(v_{\tilde{x}}-\lambda v\right), \quad \lambda \stackrel{\text { def }}{=} \frac{2}{2-q},
$$




$$
\begin{aligned}
& u_{x x}=\frac{M e^{(2-\lambda) \tilde{x}}}{\left(M^{\frac{1}{\lambda}} \rho^{\frac{q}{2}}\right)^{2}}\left[v_{\tilde{x} \tilde{x}}-(2 \lambda-1) v_{\tilde{x}}+\lambda(\lambda-1) v\right], \\
& D_{y} u=\frac{M}{\rho} e^{-\lambda \tilde{x}} D_{\tilde{y}} v .
\end{aligned}
$$

As a result, $v$ will satisfy the following differential inequality weakly:

$$
v_{\tilde{x} \tilde{x}}-(2 \lambda-1) v_{\tilde{x}}+\lambda(\lambda-1) v+\sum_{i=1}^{N-1} D_{\tilde{y}_{i}} \tilde{A}_{i}\left(\tilde{z}, v, D_{\tilde{z}} v\right) \leq 0,
$$

where we have defined

$$
\tilde{A}_{i}\left(\tilde{z}, v, D_{\tilde{z}} v\right)=\frac{\left(M^{\frac{1}{\lambda}} \rho^{\frac{q}{2}}\right)^{2}}{\rho M e^{(2-\lambda) \tilde{x}}} A_{i}(z, u, D u) .
$$

Moreover, we have

$$
\left\{\begin{array}{l}
\sum_{i=1}^{N-1} \tilde{A}_{i}\left(\tilde{z}, v, D_{\tilde{z}} v\right) \cdot D_{\tilde{y}_{i}} v \geq C_{o}\left|D_{\tilde{y}} v\right|^{q} \\
\left|\tilde{A}_{i}\left(\tilde{z}, v, D_{\tilde{z}} v\right)\right| \leq C_{1}\left|D_{\tilde{y}} v\right|^{q-1} \quad i=1, \ldots, N .
\end{array}\right.
$$

\subsection{Energy estimates for $v$}

Proposition 5 Suppose $v \geq 0$ satisfies (9) weakly in a cube $Q$ in $\mathbb{R}^{N}$. There exists a constant $\gamma>0$ depending only on the data, such that for every non-negative, piecewise smooth cutoff function $\zeta$ vanishing on $\partial$, we have for any $k \geq 0$ that

$$
\begin{aligned}
& \int_{Q}\left|D_{\tilde{x}}(v-k)_{-}\right|^{2} \zeta^{2} d \tilde{z}+\int_{Q}\left|D_{\tilde{y}}(v-k)_{-}\right|^{q} \zeta^{2} d \tilde{z} \\
& \quad \leq \gamma \int_{Q}(v-k)_{-}^{2}\left(\left|\zeta_{\tilde{x}}\right|+\left|\zeta_{\tilde{x}}\right|^{2}\right) d \tilde{z}+\gamma \int_{Q}(v-k)_{-}^{q}\left|D_{\tilde{y}} \zeta\right|^{q} d \tilde{z} .
\end{aligned}
$$

Proof For simplicity of notation, we still denote the variable of $v$ by $z=(x, y)$ and $\tilde{A}_{i}$ by $A_{i}$. In the weak formulation of (9), we take the test function $(v-k)_{-} \zeta^{2}$; discarding the non-negative contribution of $v$, a standard calculation yields that

$$
\begin{aligned}
& -\int_{Q} \zeta^{2} v_{x} D_{x}(v-k)_{-} d z-(2 \lambda-1) \int_{Q} \zeta^{2} v_{x}(v-k)_{-} d z \\
& \quad-\int_{Q} \zeta^{2} \sum_{i=1}^{N-1} A_{i}(z, v, D v) D_{y_{i}}(v-k)_{-} d z \\
& \leq 2 \int_{K} \zeta\left|v_{x}\right|(v-k)_{-}\left|\zeta_{x}\right| d z \\
& \quad+2 \int_{Q} \zeta \sum_{i=1}^{N-1}\left|A_{i}(z, v, D v)\right|(v-k)_{-}\left|D_{y_{i}} \zeta\right| d z .
\end{aligned}
$$


We will employ the structure conditions and Young's inequality repeatedly in estimating the various terms. We first estimate the terms on the left-hand side. The first term is estimated by

$$
-\int_{Q} \zeta^{2} v_{x} D_{x}(v-k)_{-} d z \geq \int_{K}\left|D_{x}(v-k)_{-}\right|^{2} \zeta^{2} d z
$$

while the second integral is

$$
\begin{aligned}
-\int_{Q} \zeta^{2} v_{x}(v-k)_{-} d z & =\frac{1}{2} \int_{K} \zeta^{2} D_{x}\left|(v-k)_{-}\right|^{2} d z \\
& =-\int_{K} \zeta \zeta_{x}\left|(v-k)_{-}\right|^{2} d z
\end{aligned}
$$

The third term is estimated by

$$
-\int_{Q} \zeta^{2} \sum_{i=1}^{N-1} A_{i}(z, v, D v) D_{y_{i}}(v-k)_{-} d z \geq C_{o} \int_{K}\left|D_{y}(v-k)_{-}\right|^{q} \zeta^{2} d z .
$$

Now we estimate the terms on the right-hand side. The first term is estimated by

$$
\begin{aligned}
& 2 \int_{Q} \zeta\left|v_{x}\right|(v-k)_{-}\left|\zeta_{x}\right| d z \\
& \quad \leq \frac{1}{2} \int_{Q} \zeta^{2}\left|D_{x}(v-k)_{-}\right|^{2} d z+2 \int_{Q}(v-k)_{-}^{2}\left|\zeta_{x}\right|^{2} d z
\end{aligned}
$$

Similarly, the second term is estimated by

$$
\begin{aligned}
& 2 \int_{Q} \zeta \sum_{i=1}^{N-1}\left|A_{i}(z, v, D v)\right|(v-k)_{-}\left|D_{y_{i}} \zeta\right| d z \\
& \quad \leq \frac{C_{O}}{2} \int_{Q} \zeta^{2}\left|D_{y}(v-k)_{-}\right|^{q} d z+\gamma \int_{Q}(v-k)_{-}^{q}\left|D_{y} \zeta\right|^{q} d z
\end{aligned}
$$

Collecting all these estimates gives the desired result.

\subsection{Shrinking the measure of the set $[v \approx 0]$}

The measure theoretical information in Lemma 5 gives that for some positive integer $j_{o}$ depending only on the data, such that

$$
\left|\left[v(\tilde{x}, \cdot) \geq 2^{-j_{o}} e^{\frac{2}{2-q} \tilde{x}}\right] \cap K_{1}\right| \geq \frac{\beta}{2}\left|K_{1}\right| \text { for all } \tilde{x} \geq 0 .
$$

Let $\tilde{x}_{o}, n_{*}>0$ to be chosen and set

$$
\bar{\epsilon}=2^{-j_{o}}, \quad k_{o}=\bar{\epsilon} e^{\frac{2}{2-q} \tilde{x}_{o}}, \quad k_{n}=\frac{k_{o}}{2^{n}} \quad \text { for } n=0,1, \ldots, n_{*} .
$$

With this stipulation the measure theoretical information above implies

$$
\left|\left[v(\tilde{x}, \cdot) \geq k_{n}\right] \cap K_{8}\right| \geq \frac{\beta}{2} \frac{1}{8^{N-1}}\left|K_{8}\right| \quad \text { for all } \tilde{x} \in\left(\tilde{x}_{o}, \infty\right),
$$

and for all $n>0$. Assume momentarily that $\tilde{x}_{o}$ has been chosen. Introduce the pair of cylinders

$$
\mathcal{Q}=\left[\tilde{x}_{o}+k_{o}^{2-q}, \tilde{x}_{o}+2 k_{o}^{2-q}\right] \times K_{8}, \quad \mathcal{Q}^{\prime}=\left[\tilde{x}_{o}, \tilde{x}_{o}+3 k_{o}^{2-q}\right] \times K_{16} .
$$


Lemma 8 There exists $n_{*}>0$ depending only on the data, such that for any $v \in(0,1)$, we have

$$
\left|\left[v \leq \frac{k_{o}}{2^{n_{*}}}\right] \cap \mathcal{Q}\right| \leq v|\mathcal{Q}| .
$$

Proof We employ the energy estimate (10) in the cylinder $\mathcal{Q}^{\prime}$, with levels $k_{n}$ and a nonnegative, piecewise smooth, cutoff function $\zeta$ in $\mathcal{Q}^{\prime}$, which equals 1 in $\mathcal{Q}$ and vanishes on $\partial \mathcal{Q}^{\prime}$, satisfying

$$
\left|D_{\tilde{y}} \zeta\right| \leq \frac{1}{8}, \quad\left|\zeta_{\tilde{x}}\right| \leq k_{o}^{q-2}
$$

Since $j_{o}$ and thus $\bar{\epsilon}$ are fixed, one verifies that if we take $\tilde{x}_{o}$ large enough then we have

$$
k_{o}^{q-2}=\bar{\epsilon}^{q-2} e^{-2 \tilde{x}_{o}} \leq 1,
$$

and as a result

$$
\left|\zeta_{\tilde{x}}\right|+\left|\zeta_{\tilde{x}}\right|^{2} \leq 2 k_{O}^{q-2}
$$

From this observation, the energy estimate (10) gives us that

$$
\begin{aligned}
\int_{\mathcal{Q}}\left|D_{\tilde{y}}\left(v-k_{n}\right)_{+}\right|^{q} d \tilde{z} & \leq \gamma k_{n}^{q}\left(1+\frac{k_{n}^{2-q}}{k_{o}^{2-q}}\right)\left|\left[v<k_{n}\right] \cap \mathcal{Q}\right| \\
& \leq \gamma k_{n}^{q}\left|\left[v<k_{n}\right] \cap \mathcal{Q}\right|,
\end{aligned}
$$

where we have used the fact that $k_{n}^{2-q} \leq k_{o}^{2-q}$.

Next, we apply [2, Chapter I, Lemma 2.2] to $v(\tilde{x}, \cdot)$ for

$$
\tilde{x} \in I \stackrel{\text { def }}{=}\left[\tilde{x}_{o}+k_{o}^{2-q}, \tilde{x}_{o}+2 k_{o}^{2-q}\right]
$$

over $K_{8}$, with levels $k_{n}>k_{n+1}$. Taking into account the measure theoretical information in (12), this gives

$$
\begin{aligned}
& \frac{k_{o}}{2^{n+1}}\left|\left[v(\tilde{x}, \cdot)<k_{n+1}\right] \cap K_{8}\right| \\
& \leq \frac{\gamma}{\left|\left[v(\tilde{x}, \cdot)>k_{n}\right] \cap K_{8}\right|} \int_{\left[k_{n+1}<v(\tilde{x}, \cdot)<k_{n}\right] \cap K_{8}}\left|D_{\tilde{y}} v\right| d \tilde{y} \\
& \quad \leq \frac{\gamma}{\beta}\left(\int_{\left[k_{n+1}<v(\tilde{x}, \cdot)<k_{n}\right] \cap K_{8}}\left|D_{\tilde{y}} v\right|^{q} d \tilde{y}\right)^{\frac{1}{q}} \\
& \quad \times\left|\left(\left[v(\tilde{x}, \cdot)<k_{n}\right]-\left[v(\tilde{x}, \cdot)<k_{n+1}\right]\right) \cap K_{8}\right|^{1-\frac{1}{q}} .
\end{aligned}
$$

Set

$$
A_{n}=\left[v<k_{n}\right] \cap \mathcal{Q}
$$

and integrate the above estimate in $d \tilde{x}$ over $I$; we obtain by using the energy estimate

$$
\begin{aligned}
\frac{k_{o}}{2^{n+1}}\left|A_{n+1}\right| & \leq \frac{\gamma}{\beta}\left(\int_{\mathcal{Q}}\left|D_{\tilde{y}}\left(v-k_{n}\right)_{+}\right|^{q} d \tilde{y}\right)^{\frac{1}{q}}\left(\left|A_{n}\right|-\left|A_{n+1}\right|\right)^{1-\frac{1}{q}} \\
& \leq \gamma \frac{k_{o}}{2^{n}}|\mathcal{Q}|^{\frac{1}{q}}\left(\left|A_{n}\right|-\left|A_{n+1}\right|\right)^{1-\frac{1}{q}} .
\end{aligned}
$$


Now take the power $\frac{q}{q-1}$ on both sides to obtain

$$
\left|A_{n+1}\right|^{\frac{q}{q-1}} \leq \gamma|\mathcal{Q}|^{\frac{1}{q-1}}\left(\left|A_{n}\right|-\left|A_{n+1}\right|\right) .
$$

Add these inequalities from 0 to $n_{*}-1$ to obtain

$$
n_{*}\left|A_{n_{*}}\right|^{\frac{q}{q-1}} \leq \sum_{n=0}^{n_{*}-1}\left|A_{n+1}\right|^{\frac{q}{q-1}} \leq \gamma|\mathcal{Q}|^{\frac{q}{q-1}} .
$$

From this we conclude that

$$
\left|A_{n_{*}}\right| \leq \frac{\gamma}{n_{*}^{\frac{q-1}{q}}}|\mathcal{Q}|
$$

Thus we may fix $n_{*}$ by choosing $\gamma n_{*}^{-\frac{q-1}{q}} \leq v$.

\subsection{A DeGiorgi-type lemma for $v$}

Suppose for the moment $\tilde{x}_{o}>0$ has been chosen in terms of the data. Assume for some $b>0$ to be determined only in terms of the data, there holds the set inclusion:

$$
\left(x_{1}, 0\right)+Q_{8}(\theta) \subset \mathcal{Q} \subset[0, b] \times K_{16}, \quad \text { where } \theta=\left(2^{-n_{*}} k_{o}\right)^{\frac{2-q}{2}}, k_{o}=\bar{\epsilon} e^{\frac{2}{2-q} \tilde{x}_{o}} .
$$

We may employ the energy estimate $(10)$ in $\left(x_{1}, 0\right)+Q_{8}(\theta)$ to obtain the following.

Lemma 9 There exists a number $v>0$ depending only upon the data, such that if

$$
\left|\left[v \leq \frac{k_{o}}{2^{n_{*}}}\right] \cap\left[\left(x_{1}, 0\right)+Q_{8}(\theta)\right]\right| \leq v\left|Q_{8}(\theta)\right|,
$$

then

$$
v \geq \frac{k_{o}}{2^{n_{*}+1}} \quad \text { a.e. in }\left(x_{1}, 0\right)+Q_{4}(\theta) .
$$

Proof We may assume $\left(x_{1}, 0\right)=(0,0)$. For $n=0,1, \ldots$, we set

$$
\begin{aligned}
l_{n} & =\frac{M_{1}}{2}+\frac{M_{1}}{2^{n+1}}, \quad M_{1}=\frac{k_{o}}{2^{n_{*}}}, \\
\rho_{n} & =4+\frac{8}{2^{n}}, \quad \tilde{\rho}_{n}=\frac{\rho_{n}+\rho_{n+1}}{2}, \\
Q_{n} & =Q_{\rho_{n}}(\theta), \quad \tilde{Q}_{n}=Q_{\tilde{\rho}_{n}}(\theta), \quad \theta=M_{1}^{\frac{2-q}{2}} .
\end{aligned}
$$

Introduce a non-negative, piecewise smooth, cutoff function $\zeta$ vanishing on $\partial Q_{n}$ and equal to identity in $\tilde{Q}_{n}$, such that

$$
\left|D_{\tilde{y}} \zeta\right| \leq \gamma 2^{n} \quad \text { and } \quad\left|\zeta_{\tilde{x}}\right| \leq \gamma \frac{2^{\frac{q}{2} n}}{\theta} .
$$

The constant $n_{*}$ will be chosen in (14) first and then $\tilde{x}_{o}$ in (17), such that

$$
\left\{\begin{array}{l}
M_{1}=\frac{e^{\frac{2}{2-q} \tilde{x}_{o}}}{2^{n_{*}}} \stackrel{\text { def }}{=} \Lambda_{o}>1 \text { and } \theta=M_{1}^{\frac{2-q}{2}} \stackrel{\text { def }}{=} \Lambda_{o}^{\frac{2-q}{2}} \\
\text { are absolute constants depending only on the data. }
\end{array}\right.
$$


Assuming this for the moment, we would have

$$
\left|\zeta_{\tilde{x}}\right|+\left|\zeta_{\tilde{x}}\right|^{2} \leq \gamma\left(\Lambda_{o}^{\frac{2-q}{2}}+\Lambda_{o}^{2-q}\right) 2^{q n} \leq \gamma \Lambda_{o}^{2-q} 2^{q n} .
$$

Consequently, the energy estimate (10) yields

$$
\begin{aligned}
& \int_{\tilde{Q}_{n}}\left|D_{\tilde{x}}\left(v-l_{n}\right)_{-}\right|^{2} d \tilde{z}+\int_{\tilde{Q}_{n}}\left|D_{\tilde{y}}\left(v-l_{n}\right)_{-}\right|^{q} d \tilde{z} \\
& \quad \leq \gamma 2^{q n} \Lambda_{o}^{2-q} \int_{Q_{n}}\left(v-l_{n}\right)_{-}^{2} d \tilde{z}+\gamma 2^{q n} \int_{Q_{n}}\left(v-l_{n}\right)_{-}^{q} d \tilde{z} \\
& \quad \leq \gamma 2^{q n} \Lambda_{o}^{2-q}\left(M_{1}^{2}+M_{1}^{q}\right)\left|A_{n}\right| \\
& \quad \leq \gamma 2^{q n} \Lambda_{o}^{4-q}\left|A_{n}\right|,
\end{aligned}
$$

where we have set $A_{n}=\left[v<l_{n}\right] \cap Q_{n}$.

Next, we may proceed exactly as in Lemma 2 to obtain the recurrence inequality

$$
Y_{n+1} \leq \gamma \Lambda_{o}^{d} \gamma_{1}^{n} Y_{n}^{1+\frac{\bar{q}}{N}} \quad \text { for } n \geq 0
$$

where $d, \gamma_{1}$ and $\gamma$ are positive constants depending only on the data, and $Y_{n}=\left|A_{n}\right| /\left|Q_{n}\right|$.

Hence by [2, Chapter I, Lemma 4.1], $Y_{n} \rightarrow 0$ if we require that

$$
Y_{o} \leq \gamma \Lambda_{o}^{-\frac{d N}{\bar{q}} \stackrel{\text { def }}{=}} v \text {. }
$$

\subsection{Expanding the positivity of $v$}

Assume momentarily that $n_{*}$ and $\tilde{x}_{o}$ have been determined. We may also assume $\bar{\epsilon} \frac{2-q}{2} e^{\tilde{x}_{o}} 2^{\frac{2-q}{2} n_{*}}$ is an integer. Then recalling the definition of $k_{o}$ and $k_{n_{*}}$ in (11), we slice $\mathcal{Q}$ into $\bar{\epsilon} \frac{2-q}{2} e^{\tilde{x}_{o}} 2^{\frac{2-q}{2} n_{*}}$ cylinders, each of length $k_{n_{*}}^{\frac{2-q}{2}}$, by setting

$$
\left\{\begin{array}{l}
Q_{n}=\left[\tilde{x}_{o}+k_{o}^{2-q}+n k_{n_{*}}^{\frac{2-q}{2}}, \tilde{x}_{o}+k_{o}^{2-q}+(n+1) k_{n_{*}}^{\frac{2-q}{2}}\right] \times K_{8} \\
\text { for } n=0,1, \ldots, \bar{\epsilon}^{\frac{2-q}{2}} e^{\tilde{x}_{o}} 2^{\frac{2-q}{2} n_{*}}-1 .
\end{array}\right.
$$

For at least one of these cylinders, say $Q_{n}$, there must hold

$$
\left|\left[v<k_{n_{*}}\right] \cap Q_{n}\right| \leq v\left|Q_{n}\right| .
$$

Having $v$ fixed in terms of the data as in Lemma 9, we may choose $n_{*}$ according to Lemma 8 , such that

$$
\gamma n_{*}^{-1} \leq v
$$

Apply Lemma 9 to $v$ over $Q_{n}$ with $\theta=k_{n_{*}}^{\frac{2-q}{2}}$ to obtain that

$$
v\left(\tilde{x}_{o}+k_{o}^{2-q}+\left(n+\frac{1}{2}\right) k_{n_{*}}^{\frac{2-q}{2}}, \cdot\right) \geq \frac{k_{n_{*}}}{2} \quad \text { a.e. in } K_{4} .
$$

Consequently, there exists some $\tilde{x}_{1}$ in the range

$$
\tilde{x}_{o}+k_{o}^{2-q}<\tilde{x}_{1}<\tilde{x}_{o}+2 k_{o}^{2-q},
$$


such that

$$
v\left(\tilde{x}_{1}, \cdot\right) \geq \sigma_{o} e^{\frac{2}{2-q} \tilde{x}_{o}} \quad \text { a.e. in } K_{4}
$$

where

$$
\sigma_{o}=\frac{\bar{\epsilon}}{2^{n_{*}+1}}
$$

\subsection{Returning to the original coordinates}

In terms of the original coordinates and the function $u$, we arrive at

$$
u\left(x_{1}, \cdot\right) \geq \sigma_{o} M e^{\frac{-2}{2-q}\left(\tilde{x}_{1}-\tilde{x}_{o}\right)} \stackrel{\text { def }}{=} M_{o} \quad \text { a.e. in } K_{4 \rho},
$$

where $x_{1}$ corresponds to $\tilde{x}_{1}$ according to the change of variables (8). Apply now Lemma 7 with $M$ replaced by $M_{O}$ to obtain that there exists $j_{*}>0$ depending only on the data, such that, setting $\epsilon=2^{-j_{*}}$,

$$
\begin{aligned}
u(x, \cdot) & \geq \epsilon M_{o}=\epsilon \sigma_{o} M e^{\frac{-2}{2-q}\left(\tilde{x}_{1}-\tilde{x}_{o}\right)} \\
& \geq \epsilon \sigma_{o} e^{\frac{-4}{2-q} e^{2 \tilde{x}_{o}}} M \quad \text { a.e. in } K_{2 \rho}
\end{aligned}
$$

for all

$$
x_{1} \leq x \leq x_{1}+\left(\epsilon M_{o}\right)^{\frac{2-q}{2}}(2 \rho)^{\frac{q}{2}} \text {. }
$$

The parameter $\tilde{x}_{o}$ is still to be chosen. Now we choose it such that the right-hand side of the above interval equals $M^{\frac{2-q}{2}} \rho^{\frac{q}{2}}$, i.e.,

$$
M^{\frac{2-q}{2}} \rho^{\frac{q}{2}} e^{-\tilde{x}_{1}}=M^{\frac{2-q}{2}} \rho^{\frac{q}{2}}-x_{1}=\left(\epsilon \sigma_{o}\right)^{\frac{2-q}{2}} M^{\frac{2-q}{2}} e^{\tilde{x}_{o}-\tilde{x}_{1}}(2 \rho)^{\frac{q}{2}},
$$

which implies the choice of $\tilde{x}_{o}$ :

$$
e^{\tilde{x}_{o}}=\frac{1}{2^{\frac{q}{2}}\left(\epsilon \sigma_{o}\right)^{\frac{2-q}{2}}} .
$$

Taking into consideration (15), this choice of $\tilde{x}_{o}$ implies that

$$
\frac{e^{\tilde{x}_{o}}}{2^{\frac{2-q}{2} n_{*}}}=\frac{2^{1-q}}{\epsilon^{\frac{2-q}{2}}} \stackrel{\text { def }}{=} \Lambda_{o}^{\frac{2-q}{2}}>1,
$$

which guarantees that (13) holds. Therefore, (16) holds for

$$
x_{1}=\rho^{\frac{q}{2}} M^{\frac{2-q}{2}}-\left(\epsilon M_{o}\right)^{\frac{2-q}{2}}(2 \rho)^{\frac{q}{2}} \leq x \leq \rho^{\frac{q}{2}} M^{\frac{2-q}{2}} .
$$

From the definition of $\tilde{x}_{o}$ and the change of variable (8) one estimates

$$
x_{1} \leq(1-\delta) M^{\frac{2-q}{2}} \rho^{\frac{q}{2}} \quad \text { where } \delta=e^{-\tilde{x}_{o}-2 e^{2 \tilde{x}_{o}}} .
$$




\subsection{Proof of Proposition 1 concluded}

Since

$$
\left|[u(\cdot, 0) \geq M] \cap K_{\rho}\right| \geq \beta\left|K_{\rho}\right|,
$$

according to Lemma 5, there exists $j_{o}>0$, such that

$$
\left|\left[u(x, \cdot) \geq \frac{M}{2^{j_{o}}}\right] \cap K_{\rho}\right| \geq \frac{\beta}{2}\left|K_{\rho}\right|
$$

for all

$$
-\left(\frac{M}{2^{j_{o}}}\right)^{\frac{2-q}{2}} \rho^{\frac{q}{2}} \leq x \leq\left(\frac{M}{2^{j_{o}}}\right)^{\frac{2-q}{2}} \rho^{\frac{q}{2}} .
$$

We apply Proposition 4 with $M$ replaced by $M_{1}=\bar{\delta} M=2^{-j_{o}} M$ and $x_{o}= \pm M_{1}^{\frac{2-q}{2}} \rho^{\frac{q}{2}}$. As a result, there exist $\eta, \delta \in(0,1)$, such that

$$
u(x, \cdot) \geq \eta \bar{\delta} M \quad \text { a.e. in } K_{2 \rho}
$$

for all

$$
|x| \leq \delta(\bar{\delta} M)^{\frac{2-q}{2}} \rho^{\frac{q}{2}} .
$$

The proof of Proposition 1 is completed by properly redefining constants $\eta$ and $\delta$.

\section{Proof of Theorem 1}

Let the parameters $\delta$ and $\eta$ be fixed as in Proposition 1 with $\beta=\frac{1}{2}$. In order to apply Proposition 1, we set

$$
\Lambda=4 \delta^{\frac{-2}{2-q}} .
$$

Then one of the following two alternatives must hold:

$$
\begin{aligned}
& \left|\left[\mu^{+}-u(0, \cdot) \geq \frac{\omega}{4}\right] \cap K_{R}\right| \geq \frac{1}{2}\left|K_{R}\right| \text { or } \\
& \left|\left[u(0, \cdot)-\mu^{-} \geq \frac{\omega}{4}\right] \cap K_{R}\right| \geq \frac{1}{2}\left|K_{R}\right| .
\end{aligned}
$$

According to Proposition 1 with $M=\frac{1}{4} \omega$, we have

$$
\pm\left(\mu^{ \pm}-u\right) \geq \frac{\eta}{4} \omega \quad \text { a.e. in } Q_{\frac{R}{2}}(\theta) \text { with } \theta=\left(\frac{\omega}{\Lambda}\right)^{\frac{2-q}{2}} .
$$

In either case, we obtain a reduction of oscillation. More precisely,

$$
\underset{Q_{\frac{R}{2}}}{\operatorname{ess}} \operatorname{osc} u \leq\left(1-\frac{\eta}{4}\right) \omega .
$$

Once we have this reduction of oscillation, the rest of the proof is quite standard. We refer to p. 45 of [2] for details. 


\section{Proof of Theorem 2 assuming Theorem 3}

Fix $z_{o} \in E$, assume $u\left(z_{o}\right)>0$, and construct cylinders

$$
z_{o}+Q_{4 \rho}(\theta) \subset E, \quad \text { where } \theta=\left[\bar{c} u\left(z_{o}\right)\right]^{\frac{2-q}{2}} .
$$

Introduce the new variables $\tilde{z}=(\tilde{x}, \tilde{y})$ defined by

$$
\tilde{y}=\frac{y-y_{o}}{\rho}, \quad \tilde{x}=\frac{x-x_{o}}{\rho^{\frac{q}{2}}\left[u\left(z_{o}\right)\right]^{\frac{2-q}{2}}} .
$$

Under this mapping, the cylinder $z_{o}+Q_{4 \rho}(\theta)$ is transformed into

$$
\tilde{Q}=\left[-4^{\frac{q}{2}} \bar{c}^{\frac{2-q}{2}}, 4^{\frac{q}{2}} \bar{c}^{\frac{2-q}{2}}\right] \times K_{4}, \quad q<2 .
$$

Consider the new function in $\tilde{Q}$ :

$$
v(\tilde{x}, \tilde{y})=\frac{1}{u\left(z_{o}\right)} u\left(x_{o}+\tilde{x} \rho^{\frac{q}{2}}\left[u\left(z_{o}\right)\right]^{\frac{2-q}{2}}, y_{o}+\rho \tilde{y}\right) .
$$

Then $v(\tilde{z})$ is a bounded non-negative, weak solution to

$$
v_{\tilde{x} \tilde{x}}+\sum_{i=1}^{N-1} D_{\tilde{y}_{i}} \tilde{A}_{i}(\tilde{z}, v, D v)=0,
$$

where the function $\tilde{A}_{i}(\tilde{z}, v, D v): E \times \mathbb{R} \times \mathbb{R}^{N} \rightarrow \mathbb{R}$ is defined by

$$
\tilde{A}_{i}(\tilde{z}, v, D v)=A_{i}(z, u, D u)\left[u\left(z_{o}\right)\right]^{1-q} \rho^{q-1},
$$

subject to the following structure conditions a.e. in $\tilde{Q}$ :

$$
\left\{\begin{array}{l}
\sum_{i=1}^{N-1} \tilde{A}_{i}(\tilde{z}, v, D v) \cdot D_{\tilde{y}_{i}} v \geq C_{o}\left|D_{\tilde{y}} v\right|^{q} \\
\left|\tilde{A}_{i}(\tilde{z}, v, D v)\right| \leq C_{1}\left|D_{\tilde{y}} v\right|^{q-1} \quad i=1, \ldots, N-1 .
\end{array}\right.
$$

Therefore Proposition 1 holds for $v$. In what follows, for simplicity of notation, let us still denote the variables of $v$ by $z=(x, y)$.

The proof of the right-hand side inequality in Theorem 2 is a consequence of the following.

Proposition 6 There exist constants $c, \bar{c} \in(0,1)$, which can be determined only in terms of the data, such that

$$
v \geq c \quad \text { in } Q_{1}(\bar{c})
$$

\subsection{Proof of Proposition 6}

For $\tau \in(0,1)$, introduce the family of nested cubes $\left\{K_{\tau}\right\}$ and the families of non-negative numbers $\left\{M_{\tau}\right\}$ and $\left\{N_{\tau}\right\}$ as follows:

$$
M_{\tau}=\sup _{K_{\tau}} v(0, \cdot), \quad N_{\tau}=(1-\tau)^{-\sigma},
$$

where $\sigma>1$ is to be chosen. The two functions $[0,1) \ni \tau \rightarrow M_{\tau}, N_{\tau}$ are increasing, and $M_{o}=N_{o}=1$ since $v(0,0)=1$. Moreover, $N_{\tau} \rightarrow \infty$ as $\tau \rightarrow 1$ whereas $M_{\tau}$ is bounded 
since $v$ is locally bounded. Therefore the equation $M_{\tau}=N_{\tau}$ has roots and we denote the largest one as $\tau_{*}$. By the continuity of $v$, there exists $\bar{y} \in K_{\tau_{*}}$, such that

$$
v(0, \bar{y})=M_{\tau_{*}}=N_{\tau_{*}}=\left(1-\tau_{*}\right)^{-\sigma} .
$$

Moreover,

$$
K_{2 R}(\bar{y}) \subset K_{\frac{1+\tau_{*}}{2}} \subset K_{1}, \quad \text { where } 2 R \stackrel{\text { def }}{=} \frac{1-\tau_{*}}{2} .
$$

Therefore by the definition of $\tau_{*}$,

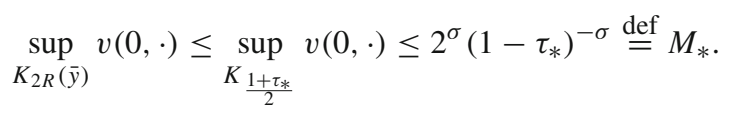

Now we apply Theorem 3 to conclude that there exists $\bar{\gamma} \in(0,1)$ depending on the data, such that

$$
\sup _{(0, \bar{y})+Q_{R}(\theta)} v \leq \bar{\gamma} M_{*} \quad \text { where } \theta=M_{*}^{\frac{2-q}{2}} .
$$

Next, we may take $\Lambda$ in the proof of Theorem 1 even larger, such that

$$
(0, \bar{y})+Q_{R}(\bar{\theta}) \subset(0, \bar{y})+Q_{R}(\theta) \quad \text { and } \underset{(0, \bar{y})+Q_{R}(\bar{\theta})}{\operatorname{osc}} v \leq \bar{\gamma} M_{*},
$$

where

$$
\bar{\theta}=\left(\frac{\bar{\gamma} M_{*}}{\Lambda}\right)^{\frac{2-q}{2}} .
$$

Then $(0, \bar{y})+Q_{R}(\bar{\theta})$ serves as the starting cylinder in Theorem 1 .

Let $\epsilon_{*} \in(0,1)$ and set $r=\epsilon_{*} R$. By Theorem 1 , for all $r<R$ and for all $z \in(0, \bar{y})+Q_{r}(\bar{\theta})$

$$
v(z)-v(0, \bar{y}) \geq-\gamma\left[\bar{\gamma} 2^{\sigma}\left(1-\tau_{*}\right)^{-\sigma}\right]\left(\frac{r}{R}\right)^{\alpha} \geq-\frac{1}{2}\left(1-\tau_{*}\right)^{-\sigma},
$$

provided we choose $\epsilon_{*}$ so small that

$$
\gamma \bar{\gamma} 2^{\sigma} \epsilon_{*}^{\alpha} \leq \frac{1}{2}
$$

This in turn gives

$$
v(z) \geq \frac{1}{2}\left(1-\tau_{*}\right) \stackrel{-\sigma}{=} \stackrel{\text { def }}{=} M \quad \text { for all } z \in(0, \bar{y})+Q_{r}(\bar{\theta}) .
$$

From this we may start employing Proposition 1 to conclude that there exist positive constants $\eta$ and $\delta$ as indicated, such that

$$
v(x, \cdot) \geq \eta M \quad \text { in } K_{2 r}(\bar{y})
$$

for all

$$
-\delta \bar{\theta} r^{\frac{q}{2}} \leq x \leq \delta \bar{\theta} r^{\frac{q}{2}}
$$

Repeated applications of Proposition 1 then yield positive constants $\eta$ and $\delta$ as indicated, such that

$$
v(x, \cdot) \geq \eta^{n} M \quad \text { in } K_{2^{n} r}(\bar{y})
$$


for all

$$
-\delta \bar{\theta} r^{\frac{q}{2}} \leq x \leq \delta \bar{\theta} r^{\frac{q}{2}}
$$

We may assume $\epsilon_{*}\left(1-\tau_{*}\right)$ is a negative, integral power of 2 . Then choose $n$ such that $2^{n} r=2$. In this way, we calculate

$$
\eta^{n} M=\frac{1}{2}\left(1-\tau_{*}\right)^{-\sigma} \eta^{n}=\frac{1}{2}\left(\frac{8}{\epsilon_{*} 2^{n}}\right)^{-\sigma} \eta^{n}=\frac{1}{2}\left(2^{\sigma} \eta\right)^{n}\left(\frac{\epsilon_{*}}{8}\right)^{\sigma} .
$$

Finally, we may choose $\sigma$ such that $2^{\sigma} \eta=1$. As a result, setting $c=2^{-3 \sigma-1} \epsilon_{*}^{\sigma}$, we have

$$
v(x, \cdot) \geq c \quad \text { in } K_{1}
$$

for all

$$
-\delta \bar{\theta} r^{\frac{q}{2}} \leq x \leq \delta \bar{\theta} r^{\frac{q}{2}}
$$

On the other hand, one estimates

$$
\begin{aligned}
\delta \bar{\theta} r^{\frac{q}{2}} & =\delta\left[\bar{\gamma} \Lambda^{-1} 2^{\sigma}\left(1-\tau_{*}\right)^{-\sigma}\right]^{\frac{2-q}{2}}\left[2^{-2} \epsilon_{*}\left(1-\tau_{*}\right)\right]^{\frac{q}{2}} \\
& =\delta^{\frac{2-q}{2}} \bar{\gamma} \frac{2-q}{2} \Lambda^{\frac{q-2}{2}} 2^{\sigma \frac{2-q}{2}-q} \epsilon_{*}^{\frac{q}{2}}\left(1-\tau_{*}\right)^{-\sigma \frac{2-q}{2}+\frac{q}{2}} \\
& \geq \delta^{\frac{2-q}{2}} \bar{\gamma}^{\frac{2-q}{2}} \Lambda^{\frac{q-2}{2}} 2^{\sigma \frac{2-q}{2}-q} \epsilon_{*}^{\frac{q}{2}} \stackrel{\text { def }}{=} \bar{c}^{\frac{2-q}{2}}
\end{aligned}
$$

provided $\sigma \geq q /(2-q)$, which may be assumed by possibly taking $\eta$ smaller if necessary. In conclusion,

$$
v \geq c \quad \text { in } Q_{1}(\bar{c})
$$

\subsection{Proof of Theorem 2 concluded}

We have shown in the last section that

$$
u\left(z_{o}\right) \leq c^{-1} \inf _{z_{o}+Q_{\rho}(\theta)} u \text { where } \theta=\left[\bar{c} u\left(z_{o}\right)\right]^{\frac{2-q}{2}} .
$$

Now we claim that

$$
\sup _{z_{o}+Q_{\rho}(\theta)} u \leq 2 c^{-1} u\left(z_{o}\right) .
$$

Indeed, if not, by continuity of $u$, there would exist $z_{*} \in z_{o}+Q_{\rho}(\theta)$ such that

$$
u\left(z_{*}\right)=2 c^{-1} u\left(z_{o}\right) .
$$

The membership of $z_{*}$ in $z_{o}+Q_{\rho}(\theta)$ implies that

$$
\left|x_{o}-x_{*}\right| \leq \rho^{\frac{q}{2}}\left[\bar{c} u\left(z_{o}\right)\right]^{\frac{2-q}{2}}=\rho^{\frac{q}{2}}\left[\frac{c \bar{c}}{2} u\left(z_{*}\right)\right]^{\frac{2-q}{2}},
$$

which in turn gives that

$$
z_{o} \in z_{*}+Q_{\bar{\rho}}\left(\theta_{*}\right) \quad \text { where } \bar{\rho}=\rho\left(\frac{c}{2}\right)^{\frac{2-q}{q}} \text { and } \theta_{*}=\left[\bar{c} u\left(z_{*}\right)\right]^{\frac{2-q}{2}} .
$$


However, this leads to a contradiction:

$$
2 c^{-1} u\left(z_{o}\right)=u\left(z_{*}\right) \leq c^{-1} \inf _{z_{*}+Q_{\bar{\rho}}\left(\theta_{*}\right)} u \leq c^{-1} u\left(z_{o}\right) .
$$

The proof of Theorem 2 is now concluded by properly redefining $c$.

\section{Proof of Theorem 3}

We first present two propositions from which Theorem 3 follows.

Proposition 7 Let u be a non-negative, local, weak super-solution to (1) and (2) in E. There exists a constant $\gamma>0$ depending only on the data, such that for all cylinders

$$
\left[x_{o}-T, x_{o}+T\right] \times K_{2 \rho}\left(y_{o}\right) \subset E,
$$

we have

$$
\sup _{0<t<T} \frac{1}{t \rho^{N-1}} \int_{x_{o}-t}^{x_{o}+t} \int_{K_{\rho}\left(y_{o}\right)} u d y d x \leq \frac{\gamma}{\rho^{N-1}} \int_{K_{2 \rho}\left(y_{o}\right)} u\left(x_{o}, y\right) d y+\gamma\left(\frac{T^{2}}{\rho^{q}}\right)^{\frac{1}{2-q}} .
$$

Proposition 8 Let $u$ be a locally bounded, local, weak sub(super)-solution to (1) and (2) in E. For $r \in(0,2]$, let

$$
\kappa_{r} \stackrel{\text { def }}{=}(N+r-1) q-2(N-1)>0 .
$$

There exists a constant $\gamma>0$ depending only on the data, such that for all cylinders

$$
\left[x_{o}-2 t, x_{o}+2 t\right] \times K_{2 \rho}\left(y_{o}\right) \subset E,
$$

we have

$$
\begin{aligned}
\underset{\left[x_{o}-t, x_{o}+t\right] \times K_{\rho}\left(y_{o}\right)}{\operatorname{ess} \sup _{ \pm}} & \leq \gamma\left(\frac{\rho^{q}}{t^{2}}\right)^{\frac{N-1}{\kappa_{r}}}\left(f_{K_{2 \rho}\left(y_{o}\right)} f_{x_{o}-2 t}^{x_{o}+2 t} u_{ \pm}^{r} d x d y\right)^{\frac{q}{\kappa_{r}}} \\
& +\gamma\left(\frac{t^{2}}{\rho^{q}}\right)^{\frac{1}{2-q}} .
\end{aligned}
$$

\subsection{Proof of Proposition 7}

Assuming $z_{o}=(0,0)$, let us consider the cylinder $[-T, T] \times K_{\rho}$. In what follows we denote by $\zeta_{2}(y)$ a piecewise smooth function that vanishes on $K_{\rho}$ and equals 1 on $K_{\sigma \rho}$, such that

$$
\left|D_{y} \zeta_{2}\right| \leq \frac{\gamma}{(1-\sigma) \rho},
$$

whereas for $0<t<T$ we define

$$
\zeta_{1}(x)=\left(1-\frac{x^{2}}{t^{2}}\right)_{+} .
$$

Let us introduce a constant $\alpha$ that satisfies

$$
1<\alpha<\min \left\{q, \frac{1}{q-1}\right\}
$$


such that the quantities $2-\alpha, q-\alpha$ and $\alpha(q-1)$ are all in $(0,1)$. The proof of Proposition 7 replies on the following two lemmas.

\subsubsection{Auxiliary lemmas}

Lemma 10 Suppose the hypothesis in Proposition 7 holds. There exists a constant $\gamma>0$ depending only on the data and $\alpha$, such that

$$
\begin{aligned}
& \int_{-t}^{t} \int_{K_{\rho}}\left|D_{y} u\right|^{q} u^{-\alpha} \zeta_{1} \zeta_{2}^{q} d y d x \\
& \quad \leq \frac{\gamma}{t^{2}} \int_{-t}^{t} \int_{K_{\rho}} u^{2-\alpha} d y d x+\frac{\gamma}{(1-\sigma)^{q} \rho^{q}} \int_{-t}^{t} \int_{K_{\rho}} u^{q-\alpha} d y d x
\end{aligned}
$$

Proof We may assume that $u \geq \epsilon>0$ for otherwise we may work with $u+\epsilon$ and then let $\epsilon \rightarrow 0$. Now we use $u^{1-\alpha} \zeta_{1} \zeta_{2}^{q}$ as a test function in the weak formulation of (1). Formally, we have

$$
\int_{-t}^{t} \int_{K_{\rho}} u_{x x} u^{1-\alpha} \zeta_{1} \zeta_{2}^{q} d y d x+\sum_{i=1}^{N-1} \int_{-t}^{t} \int_{K_{\rho}} D_{y_{i}} A_{i}(z, u, D u) u^{1-\alpha} \zeta_{1} \zeta_{2}^{q} d y d x \leq 0
$$

We estimate the two terms on the left separately. First of all,

$$
\begin{aligned}
& \int_{-t}^{t} \int_{K_{\rho}} u_{x x} u^{1-\alpha} \zeta_{1} \zeta_{2}^{q} d y d x \\
& \quad=(\alpha-1) \int_{-t}^{t} \int_{K_{\rho}}\left|u_{x}\right|^{2} u^{-\alpha} \zeta_{1} \zeta_{2}^{q} d y d x+\frac{2}{t^{2}} \int_{-t}^{t} \int_{K_{\rho}} x u_{x} u^{1-\alpha} \zeta_{2}^{q} d y d x \\
& \quad=(\alpha-1) \int_{-t}^{t} \int_{K_{\rho}}\left|u_{x}\right|^{2} u^{-\alpha} \zeta_{1} \zeta_{2}^{q} d y d x+\frac{2}{(2-\alpha) t^{2}} \int_{-t}^{t} \int_{K_{\rho}} x D_{x}\left(u^{2-\alpha}\right) \zeta_{2}^{q} d y d x \\
& \quad \geq(\alpha-1) \int_{-t}^{t} \int_{K_{\rho}}\left|u_{x}\right|^{2} u^{-\alpha} \zeta_{1} \zeta_{2}^{q} d y d x-\frac{2}{(2-\alpha) t^{2}} \int_{-t}^{t} \int_{K_{\rho}} u^{2-\alpha} \zeta_{2}^{q} d y d x .
\end{aligned}
$$

Next,

$$
\begin{aligned}
& \sum_{i=1}^{N-1} \int_{-t}^{t} \int_{K_{\rho}} D_{y_{i}} A_{i}(z, u, D u) u^{1-\alpha} \zeta_{1} \zeta_{2}^{q} d y d x \\
& \geq C_{o}(\alpha-1) \int_{-t}^{t} \int_{K_{\rho}}\left|D_{y} u\right|^{q} u^{-\alpha} \zeta_{1} \zeta_{2}^{q} d y d x \\
& \quad-q C_{1} \int_{-t}^{t} \int_{K_{\rho}}\left|D_{y} u\right|^{q-1} u^{1-\alpha} \zeta_{1} \zeta_{2}^{q-1}\left|D_{y} \zeta_{2}\right| d y d x \\
& \geq \frac{C_{o}}{2}(\alpha-1) \int_{-t}^{t} \int_{K_{\rho}}\left|D_{y} u\right|^{q} u^{-\alpha} \zeta_{1} \zeta_{2}^{q} d y d x \\
& \quad-\gamma \int_{-t}^{t} \int_{K_{\rho}} u^{q-\alpha}\left|D_{y} \zeta_{2}\right|^{q} d y d x
\end{aligned}
$$

Combining them we obtain the desired conclusion. 
Lemma 11 Suppose the hypothesis in Proposition 7 holds. There exists a constant $\gamma>0$ depending only on the data and $\alpha$, such that

$$
\frac{1}{(1-\sigma) \rho^{N}} \int_{-t}^{t} \int_{K_{\rho}}\left|D_{y} u\right|^{q-1} \zeta_{1} \zeta_{2}^{q-1} d y d x \leq \frac{\gamma t^{\frac{2-q}{q}}}{(1-\sigma) \rho} \mathcal{S}^{2 \frac{q-1}{q}}+\frac{\gamma t}{(1-\sigma)^{q} \rho^{q}} \mathcal{S}^{q-1},
$$

where

$$
\mathcal{S}=\sup _{0<t<T} \frac{1}{t \rho^{N-1}} \int_{-t}^{t} \int_{K_{\rho}} u d y d x .
$$

Proof Notice that by our choice of $\alpha$, the quantities $2-\alpha, q-\alpha$ and $\alpha(q-1)$ are all in $(0,1)$. By Hölder's inequality and Lemma 10, we estimate

$$
\begin{aligned}
& \frac{1}{(1-\sigma) \rho^{N}} \int_{-t}^{t} \int_{K_{\rho}}\left|D_{y} u\right|^{q-1} \zeta_{1} \zeta_{2}^{q-1} d y d x \\
& \leq \frac{1}{(1-\sigma) \rho^{N}}\left[\int_{-t}^{t} \int_{K_{\rho}}\left|D_{y} u\right|^{q} u^{-\alpha} \zeta_{1} \zeta_{2}^{q} d y d x\right]^{\frac{q-1}{q}}\left[\int_{-t}^{t} \int_{K_{\rho}} u^{\alpha(q-1)} d y d x\right]^{\frac{1}{q}} \\
& \leq \frac{1}{(1-\sigma) \rho^{N}}\left[\frac{\gamma}{(1-\sigma)^{q} \rho^{q}} \int_{-t}^{t} \int_{K_{\rho}} u^{q-\alpha} d y d x\right]^{\frac{q-1}{q}}\left[\int_{-t}^{t} \int_{K_{\rho}} u^{\alpha(q-1)} d y d x\right]^{\frac{1}{q}} \\
& \quad+\frac{1}{(1-\sigma) \rho^{N}}\left[\frac{\gamma}{t^{2}} \int_{-t}^{t} \int_{K_{\rho}} u^{2-\alpha} d y d x\right]^{\frac{q-1}{q}}\left[\int_{-t}^{t} \int_{K_{\rho}} u^{\alpha(q-1)} d y d x\right]^{\frac{1}{q}} \\
& \leq \frac{\gamma t^{\frac{2-q}{q}}}{(1-\sigma) \rho} \mathcal{S}^{\frac{q-1}{q}}+\frac{\gamma t}{(1-\sigma)^{q} \rho^{q}} \mathcal{S}^{q-1} .
\end{aligned}
$$

\subsubsection{Proof of Proposition 7 concluded}

We use $\zeta_{1} \zeta_{2}^{q}$ as a test function in the weak formulation of (1) for super-solutions. Formally, we have

$$
\int_{-t}^{t} \int_{K_{\rho}} u_{x x} \zeta_{1} \zeta_{2}^{q} d y d x+\sum_{i=1}^{N-1} \int_{-t}^{t} \int_{K_{\rho}} D_{y_{i}} A_{i}(z, u, D u) \zeta_{1} \zeta_{2}^{q} d y d x \leq 0 .
$$

We estimate the two terms on the left separately. First of all,

$$
\begin{aligned}
& \int_{-t}^{t} \int_{K_{\rho}} u_{x x} \zeta_{1} \zeta_{2}^{q} d y d x=\frac{2}{t^{2}} \int_{-t}^{t} \int_{K_{\rho}} x u_{x} \zeta_{2}^{q} d y d x \\
& \quad=\frac{2}{t} \int_{K_{\rho}} u(t, \cdot) \zeta_{2}^{q} d y+\frac{2}{t} \int_{K_{\rho}} u(-t, \cdot) \zeta_{2}^{q} d y-\frac{2}{t^{2}} \int_{-t}^{t} \int_{K_{\rho}} u \zeta_{2}^{q} d y d x \\
& \quad=2 \rho^{N-1} h^{\prime}(t),
\end{aligned}
$$

where

$$
h(t)=\frac{1}{t \rho^{N-1}} \int_{-t}^{t} \int_{K_{\rho}} u \zeta_{2}^{q} d y d x
$$


On the other hand, we estimate

$$
\begin{aligned}
\int_{-t}^{t} \int_{K_{\rho}} D_{y_{i}} A_{i}(z, u, D u) \zeta_{1} \zeta_{2}^{q} d y d x & =-q \int_{-t}^{t} \int_{K_{\rho}} A_{i}(z, u, D u) \zeta_{1} D_{y_{i}} \zeta_{2} \zeta_{2}^{q-1} d y d x \\
& \geq-\frac{q C_{1}}{(1-\sigma) \rho} \int_{-t}^{t} \int_{K_{\rho}}\left|D_{y} u\right|^{q-1} \zeta_{1} \zeta_{2}^{q-1} d y d x
\end{aligned}
$$

Combining the above two estimates we obtain

$$
h^{\prime}(t) \leq \frac{\gamma}{(1-\sigma) \rho^{N}} \int_{-t}^{t} \int_{K_{\rho}}\left|D_{y} u\right|^{q-1} \zeta_{1} \zeta_{2}^{q-1} d y d x .
$$

We integrate the above inequality in $d t$ over $(0, \tau)$ for $\tau<T$, use Lemma 11 and apply Young's inequality to obtain for an arbitrary $\delta \in(0,1)$,

$$
\begin{aligned}
h(\tau)-h(0) & \leq \gamma \frac{\tau^{\frac{2}{q}}}{(1-\sigma) \rho} \mathcal{S}^{2 \frac{q-1}{q}}+\gamma \frac{\tau^{2}}{(1-\sigma)^{q} \rho^{q}} \mathcal{S}^{q-1} \\
& \leq \delta \mathcal{S}+\frac{\gamma}{(1-\sigma)^{\frac{q}{2-q}} \delta^{\frac{q-1}{2-q}}}\left(\frac{T^{2}}{\rho^{q}}\right)^{\frac{1}{2-q}} .
\end{aligned}
$$

As $\tau$ ranges over $(0, T)$, we arrive at

$$
\begin{gathered}
\sup _{0<t<T} \frac{1}{t \rho^{N-1}} \int_{-t}^{t} \int_{K_{\sigma \rho}} u d y d x \leq \delta \sup _{0<t<T} \frac{1}{t \rho^{N-1}} \int_{-t}^{t} \int_{K_{\rho}} u d y d x \\
+\frac{2}{\rho^{N-1}} \int_{K_{\rho}} u(0, y) d y+\frac{\gamma}{(1-\sigma)^{\frac{q}{2-q}} \delta^{\frac{q-1}{2-q}}}\left(\frac{T^{2}}{\rho^{q}}\right)^{\frac{1}{2-q}} .
\end{gathered}
$$

An interpolation argument ([2, Chapter I, Lemma 4.3]) yields that

$$
\sup _{0<t<T} \frac{1}{t \rho^{N-1}} \int_{-t}^{t} \int_{K_{\rho}} u d y d x \leq \frac{\gamma}{\rho^{N-1}} \int_{K_{2 \rho}} u(0, y) d y+\gamma\left(\frac{T^{2}}{\rho^{q}}\right)^{\frac{1}{2-q}} .
$$

\subsection{Proof of Proposition 8}

For parameters $k, \rho, t>0$ and $n=0,1, \ldots$, we set

$$
\begin{aligned}
& k_{n}=k-\frac{k}{2^{n}}, \quad \rho_{n}=\sigma \rho+\frac{(1-\sigma) \rho}{2^{n}}, \quad \tilde{\rho}_{n}=\frac{\rho_{n}+\rho_{n+1}}{2}, \\
& t_{n}=\sigma t+\frac{(1-\sigma) t}{2^{n}}, \quad \tilde{t}_{n}=\frac{t_{n}+t_{n+1}}{2}, \\
& Q_{n}=\left[-t_{n}, t_{n}\right] \times K_{\rho_{n}}, \quad \tilde{Q}_{n}=\left[-\tilde{t}_{n}, \tilde{t}_{n}\right] \times K_{\tilde{\rho}_{n}} .
\end{aligned}
$$

By construction $Q_{\infty}=[-\sigma t, \sigma t] \times K_{\sigma \rho}$. Assuming $u$ is locally bounded in $E$, we may define

$$
M=\underset{Q_{o}}{\operatorname{ess} \sup } u_{+}, \quad M_{\sigma}=\underset{Q_{\infty}}{\operatorname{ess} \sup } u_{+} .
$$

Introduce the cutoff function $\zeta$ vanishing on $\partial Q_{n}$ and equal to identity in $\tilde{Q}_{n}$, such that

$$
\left|D_{y} \zeta\right| \leq \frac{\gamma 2^{n}}{(1-\sigma) \rho} \quad \text { and } \quad\left|D_{x} \zeta\right| \leq \frac{\gamma 2^{n}}{(1-\sigma) t} .
$$


In this setting the energy estimate in Proposition 2 yields

$$
\begin{aligned}
& \int_{\tilde{Q}_{n}}\left|D_{x}\left(u-k_{n+1}\right)_{+}\right|^{2} d z+\int_{\tilde{Q}_{n}}\left|D_{y}\left(u-k_{n+1}\right)_{+}\right|^{q} d z \\
& \quad \leq \frac{\gamma 2^{2 n}}{(1-\sigma)^{2} t^{2}} \int_{Q_{n}}\left(u-k_{n+1}\right)_{+}^{2} d z+\frac{\gamma 2^{q n}}{(1-\sigma)^{q} \rho^{q}} \int_{Q_{n}}\left(u-k_{n+1}\right)_{+}^{q} d z .
\end{aligned}
$$

Next setting $A_{n}=\left[u>k_{n+1}\right] \cap Q_{n}$, we observe that for any $r>0$

$$
\int_{Q_{n}}\left(u-k_{n}\right)_{+}^{r} d z \geq\left(k_{n+1}-k_{n}\right)^{r}\left|\left[u>k_{n+1}\right] \cap Q_{n}\right|=\frac{k^{r}}{2^{r(n+1)}}\left|A_{n}\right| .
$$

As a result, applying Hölder's inequality and the above observation with $r=2$, we estimate the second integral on the right-hand side of the energy estimate by

$$
\begin{aligned}
\int_{Q_{n}}\left(u-k_{n+1}\right)_{+}^{q} d z & \leq\left(\int_{Q_{n}}\left(u-k_{n+1}\right)_{+}^{2} d z\right)^{\frac{q}{2}}\left|\left[u>k_{n+1}\right] \cap Q_{n}\right|^{1-\frac{q}{2}} \\
& \leq \frac{\gamma 2^{2 n-q n}}{k^{2-q}} \int_{Q_{n}}\left(u-k_{n}\right)_{+}^{2} d z .
\end{aligned}
$$

Putting this back to the energy estimate gives us

$$
\begin{aligned}
& \int_{\tilde{Q}_{n}}\left|D_{x}\left(u-k_{n+1}\right)_{+}\right|^{2} d z+\int_{\tilde{Q}_{n}}\left|D_{y}\left(u-k_{n+1}\right)_{+}\right|^{q} d z \\
& \quad \leq \frac{\gamma 2^{2 n} k^{q-2}}{(1-\sigma)^{2} \rho^{q}}\left(1+k^{2-q} \frac{\rho^{q}}{t^{2}}\right) \int_{Q_{n}}\left(u-k_{n}\right)_{+}^{2} d z .
\end{aligned}
$$

Now setting $\zeta$ to be a cutoff function which vanishes on the boundary of $\tilde{Q}_{n}$ and equals identity in $Q_{n+1}$, an application of Lemma 1 and the energy estimate gives that

$$
\begin{aligned}
& \int_{Q_{n+1}}\left(u-k_{n+1}\right)_{+}^{2} d z \leq \int_{\tilde{Q}_{n}}\left(u-k_{n+1}\right)_{+}^{2} \zeta^{2} d z \\
& \leq\left(\int_{\tilde{Q}_{n}}\left[\left(u-k_{n+1}\right)_{+} \zeta\right]^{\frac{N \bar{q}}{N-\bar{q}}} d z\right)^{2 \frac{N-\bar{q}}{N \bar{q}}}\left|A_{n}\right|^{1-\frac{2}{\bar{q}}+\frac{2}{N}} \\
& \leq \gamma\left(\left\|D_{x}\left(u-k_{n+1}\right)_{+}\right\|_{2} \prod_{i=1}^{N-1}\left\|D_{y_{i}}\left(u-k_{n+1}\right)_{+}\right\|_{q}\right)^{\frac{2}{N}}\left|A_{n}\right|^{1-\frac{2}{\bar{q}}+\frac{2}{N}} \\
& \leq\left[\frac{\gamma 2^{2 n} k^{q-2}}{(1-\sigma)^{2} \rho^{q}}\left(1+k^{2-q} \frac{\rho^{q}}{t^{2}}\right)\right]^{\frac{2}{\bar{q}}}\left(\frac{2^{2 n}}{k^{2}}\right)^{1-\frac{2}{\bar{q}}+\frac{2}{N}}\left[\int_{Q_{n}}\left(u-k_{n}\right)_{+}^{2} d z\right]^{1+\frac{2}{N}},
\end{aligned}
$$

where $\bar{q}$ satisfies

$$
\frac{1}{\bar{q}}=\frac{1}{N}\left(\frac{1}{2}+\frac{N-1}{q}\right)
$$

Hence by setting

$$
Y_{n}=f_{Q_{n}}\left(u-k_{n}\right)_{+}^{2} d z
$$


we arrive at the recurrence inequality

$$
Y_{n+1} \leq \frac{\gamma \gamma_{1}^{n}}{(1-\sigma)^{\frac{4}{q}} k^{\frac{4}{N}}}\left(\frac{1}{k^{2-q}} \frac{t^{2}}{\rho^{q}}\right)^{\frac{1}{N}}\left(1+k^{2-q} \frac{\rho^{q}}{t^{2}}\right)^{\frac{2}{\bar{q}}} Y_{n}^{1+\frac{2}{N}},
$$

where $\gamma, \gamma_{1}>0$ are absolute constants.

Now let us stipulate that

$$
k \geq\left(\frac{t^{2}}{\rho^{q}}\right)^{\frac{1}{2-q}}
$$

such that the above recurrence inequality becomes

$$
\begin{aligned}
Y_{n+1} & \leq \frac{\gamma \gamma_{1}^{n}}{(1-\sigma)^{\frac{4}{q}} k^{\frac{4}{N}}}\left(\frac{1}{k^{2-q}} \frac{t^{2}}{\rho^{q}}\right)^{\frac{1}{N}}\left(k^{2-q} \frac{\rho^{q}}{t^{2}}\right)^{\frac{2}{\bar{q}}} Y_{n}^{1+\frac{2}{N}} \\
& \leq \frac{\gamma \gamma_{1}^{n}}{(1-\sigma)^{\frac{4}{\bar{q}}} k^{\frac{4}{N}}}\left(k^{2-q} \frac{\rho^{q}}{t^{2}}\right)^{\frac{2}{\bar{q}}-\frac{1}{N}} Y_{n}^{1+\frac{2}{N}} \\
& \leq \frac{\gamma \gamma_{1}^{n}}{(1-\sigma)^{\frac{4}{\bar{q}}} k^{\frac{4}{N}}}\left(k^{2-q} \frac{\rho^{q}}{t^{2}}\right)^{\frac{2(N-1)}{N q}} Y_{n}^{1+\frac{2}{N}} .
\end{aligned}
$$

Hence by [2, Chapter I, Lemma 4.1], $Y_{n} \rightarrow 0$, i.e. $M_{\sigma} \leq k$, if we require that

$$
Y_{o} \leq \gamma(1-\sigma)^{\frac{2 N}{\bar{q}}} k^{2}\left(k^{2-q} \frac{\rho^{q}}{t^{2}}\right)^{-\frac{N-1}{q}}=\gamma(1-\sigma)^{\frac{2 N}{\bar{q}}} k^{\frac{k_{2}}{q}}\left(\frac{t^{2}}{\rho^{q}}\right)^{\frac{N-1}{q}} .
$$

This is fulfilled if we require $\kappa_{2}=(N+1) q-2(N-1)>0$ and take

$$
k=\frac{\gamma Y_{o}^{\frac{q}{\kappa_{2}}}}{(1-\sigma)^{\frac{2 q N}{\kappa_{2} \bar{q}}}}\left(\frac{\rho^{q}}{t^{2}}\right)^{\frac{N-1}{\kappa_{2}}}+\left(\frac{t^{2}}{\rho^{q}}\right)^{\frac{1}{2-q}} .
$$

As a result, we arrive at

$$
\begin{aligned}
M_{\sigma} & \leq \frac{\gamma}{(1-\sigma)^{\frac{2 q N}{\kappa_{2} \tilde{q}}}}\left(\frac{\rho^{q}}{t^{2}}\right)^{\frac{N-1}{\kappa_{2}}}\left(f_{Q_{o}} u_{+}^{2} d z\right)^{\frac{q}{\kappa_{2}}}+\left(\frac{t^{2}}{\rho^{q}}\right)^{\frac{1}{2-q}} \\
& \leq \frac{\gamma M^{\frac{q(2-r)}{\kappa_{2}}}}{(1-\sigma)^{\frac{2 q N}{\kappa_{2} \tilde{q}}}}\left(\frac{\rho^{q}}{t^{2}}\right)^{\frac{N-1}{\kappa_{2}}}\left(f_{Q_{o}} u_{+}^{r} d z\right)^{\frac{q}{\kappa_{2}}}+\left(\frac{t^{2}}{\rho^{q}}\right)^{\frac{1}{2-q}},
\end{aligned}
$$

for any $r$ satisfying $r<2$ and $\kappa_{r}=(N+r-1) q-2(N-1)>0$. Thus we have

$$
0<\frac{q(2-r)}{\kappa_{2}}=1-\frac{\kappa_{r}}{\kappa_{2}}<1,
$$

and an interpolation argument ([2, Chapter I, Lemma 4.3]) gives that

$$
\operatorname{ess}_{[-t, t] \times K_{\rho}} u_{+} \leq \gamma\left(\frac{\rho^{q}}{t^{2}}\right)^{\frac{N-1}{\kappa_{r}}}\left(f_{K_{2 \rho}} f_{-2 t}^{2 t} u_{+}^{r} d x d y\right)^{\frac{q}{\kappa_{r}}}+\gamma\left(\frac{t^{2}}{\rho^{q}}\right)^{\frac{1}{2-q}} .
$$


Acknowledgements Open access funding provided by Paris Lodron University of Salzburg. We would like to acknowledge the support from INdAM-INGV joint strategic project SIES which funded the CIME-EMS Summer School in Applied Mathematics, held in Cetraro, July 2019. Our collaboration on this project started from this meeting. We would also thank K. Buryachenko and S. Ciani for interesting conversations during this meeting. N. Liao is supported by the FWF-Project 35 P31956-N32 "Doubly nonlinear evolution equations". $\mathrm{V}$. Vespri is a member of GNAMPA (INdAM). The authors are grateful to the anonymous referees for careful reading and helpful comments.

Open Access This article is licensed under a Creative Commons Attribution 4.0 International License, which permits use, sharing, adaptation, distribution and reproduction in any medium or format, as long as you give appropriate credit to the original author(s) and the source, provide a link to the Creative Commons licence, and indicate if changes were made. The images or other third party material in this article are included in the article's Creative Commons licence, unless indicated otherwise in a credit line to the material. If material is not included in the article's Creative Commons licence and your intended use is not permitted by statutory regulation or exceeds the permitted use, you will need to obtain permission directly from the copyright holder. To view a copy of this licence, visit http://creativecommons.org/licenses/by/4.0/.

\section{References}

1. Cupini, G., Marcellini, P., Mascolo, E.: Regularity of minimizers under limit growth conditions. Nonlinear Anal. 153, 294-310 (2017)

2. DiBenedetto, E.: Degenerate Parabolic Equations. Universitext. Springer, New York (1993)

3. DiBenedetto, E.: Partial Differential Equations, 2nd edn. Birkhäuser, Boston (2009)

4. DiBenedetto, E., Gianazza, U., Vespri, V.: Harnack estimates for quasi-linear degenerate parabolic differential equations. Acta Math. 200(2), 181-209 (2008)

5. DiBenedetto, E., Gianazza, U., Vespri, V.: Forward, backward and elliptic Harnack inequality for nonnegative solutions to a certain singular parabolic partial differential equations. Ann. Scuola Norm. Sup. Pisa Cl. Sci. (5) 9(2), 385-422 (2010)

6. DiBenedetto, E., Gianazza, U., Vespri, V.: A new approach to the expansion of positivity set of nonnegative solutions to certain singular parabolic partial differential equations. Proc. Am. Math. Soc. 138(10), 3521-3529 (2010)

7. DiBenedetto, E., Gianazza, U., Vespri, V.: Harnack's Inequality for Degenerate and Singular Parabolic Equations, Springer Monographs in Mathematics. Springer, New York (2012)

8. DiBenedetto, E., Gianazza, U., Vespri, V.: Remarks on local boundedness and local Hölder continuity of local weak solutions to anisotropic p-Laplacian type equations. J. Elliptic Parabol. Equ. 2(1-2), 157-169 (2016)

9. Düzgün, F.G., Marcellini, P., Vespri, V.: Space expansion for a solution of an anisotropic p-Laplacian equation by using a parabolic approach. Riv. Math. Univ. Parma 5, 93-111 (2014)

10. Fusco, N., Sbordone, C.: Local boundedness of minimizers in a limit case. Manuscr. Math. 69(1), 19-25 (1990)

11. Giaquinta, M.: Growth conditions and regularity, a counter example. Manuscr. Math. 59(2), 245-248 (1987)

12. Kolodii, I.M.: The boundedness of generalized solutions of elliptic differential equations. Vestnik Moskov. Univ. Ser. I Mat. Meh. 25(5), 44-52 (1970) (in Russian). English transl.: Moscow Univ. Math. Bull. 25(5), 31-37 (1970)

13. Ladyzhenskaya, O.A., Ural'tseva, N.N.: Linear and Quasilinear Elliptic Equations. Academic Press, New York (1968)

14. Lieberman, G.M.: Gradient estimates for a new class of degenerate elliptic and parabolic equations. Ann. Scuola Norm. Sup. Pisa Cl. Sci. (4) 21(4), 497-522 (1994)

15. Lieberman, G.M.: Gradient estimates for anisotropic elliptic equations. Adv. Differ. Equ. 10(7), 767-812 (2005)

16. Liskevich, V., Skrypnik, I.I.: Hölder continuity of solutions to an anisotropic elliptic equation. Nonlinear Anal. 71(5-6), 1699-1708 (2009)

17. Marcellini, P.: Un exemple de solution discontinue d'un probléme variationel dans le cas scalaire. Preprint 11, Ist. Mat. "U. Dini", Firenze (1987-1988)

18. Marcellini, P.: Regularity of minimizers of integrals of the calculus of variations with nonstandard growth conditions. Arch. Ration. Mech. Anal. 105(3), 267-284 (1989) 
19. Marcellini, P.: Regularity and existence of solutions of elliptic equations with $p, q$-growth conditions. J. Differ. Equ. 90(1), 1-30 (1991)

20. Marcellini, P.: Regularity for elliptic equations with general growth conditions. J. Differ. Equ. 105(2), 296-333 (1993)

21. Moser, J.: On Harnack's theorem for elliptic differential equations. Commun. Pure Appl. Math. 14, 577$591(1961)$

22. Serrin, J.: Local behavior of solutions of quasi-linear equations. Acta Math. 111(1), 247-302 (1964)

23. Troisi, M.: Teoremi di inclusione per spazi di Sobolev non isotropi. Ric. Mat. 18, 3-24 (1969)

24. Trudinger, N.: On Harnack type inequalities and their application to quasilinear elliptic equations. Commun. Pure Appl. Math. 20, 721-747 (1967)

Publisher's Note Springer Nature remains neutral with regard to jurisdictional claims in published maps and institutional affiliations. 